\title{
Relaxed Stratification: A New Approach to Practical Complete Predicate Refinement*
}

\author{
Tachio Terauchi ${ }^{1}$ and Hiroshi Unno ${ }^{2}$ \\ 1 JAIST \\ terauchi@jaist.ac.jp \\ 2 University of Tsukuba \\ uhiro@cs.tsukuba.ac.jp
}

\begin{abstract}
In counterexample-guided abstraction refinement, a predicate refinement scheme is said to be complete for a given theory if it is guaranteed to eventually find predicates sufficient to prove the given property, when such exist. However, existing complete methods require deciding if a proof of the counterexample's spuriousness exists in some finite language of predicates. Such an exact finite-language-restricted predicate search is quite hard for many theories used in practice and incurs a heavy overhead. In this paper, we address the issue by showing that the language restriction can be relaxed so that the refinement process is restricted to infer proofs from some finite language $L_{\text {base }} \cup L_{\text {ext }}$ but is only required to return a proof when the counterexample's spuriousness can be proved in $L_{\text {base }}$. Then, we show how a proof-based refinement algorithm can be made to satisfy the relaxed requirement and be complete by restricting only the theory-level reasoning in SMT to emit

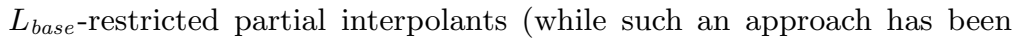
proposed previously, we show for the first time that it can be done for languages that are not closed under conjunctions and disjunctions). We also present a technique that uses a property of counterexample patterns to further improve the efficiency of the refinement algorithm while still satisfying the requirement. We have experimented with a prototype implementation of the new refinement algorithm, and show that it is able to achieve complete refinement with only a small overhead.
\end{abstract}

\section{Introduction}

Predicate abstraction with counterexample-guided abstraction refinement $(\mathrm{CE}-$ GAR) is a promising approach to automated verification of safety (i.e., reachability) properties (see, e.g., [7] for a survey). Briefly, the CEGAR approach works as follows. Let $\mathcal{T}$ be a first-order logic (FOL) theory. The verifier picks some finite set of predicates from $\mathcal{T}$ as the initial candidate predicate set, and iterates the following two processes until convergence (here, we use the term "predicate" for an arbitrary formula, and not limited to just atomic predicates).

* This work was supported by MEXT Kakenhi 23220001, 26330082, 25280023, and 25730035. 
(1) The abstraction process checks if the current candidates form a sufficient proof of the program's safety (i.e., an inductive invariant - sometimes called "safe" inductive invariant). If so, then the program is proved safe and the iteration halts. Otherwise, the process generates a counterexample as an evidence that the current candidates are insufficient, and (2) is invoked.

(2) The refinement process analyzes the given counterexample. If the counterexample cannot be proved spurious by predicates from $\mathcal{T}$ (i.e., "the counterexample is real"), then the iteration halts and the program is detected to be unsafe. Otherwise, the predicates inferred as a proof of the counterexample's spuriousness are added to the candidates, and we repeat from (1).

Note that the verifier halts either when sufficient predicates are inferred to prove the program safe, or a real counterexample is discovered.

For an unsafe program, the state-of-the-art CEGAR-based verifiers are usually able to eventually discover a real counterexample and converge, by exploring the state space in a fair manner (if somewhat slowly for ones requiring large counterexamples). By contrast, when a program is safe and the underlying theory $\mathcal{T}$ is sufficient for proving the safety, most verifiers have no guarantee of convergence and can diverge by having the refinement process indefinitely produce incorrect candidate proofs.

For example, consider the C-like program shown in Figure 1. Here, ndet() returns a non-deterministic integer. The goal is to verify that the assertion failure is unreachable, that is, $a=b \Rightarrow y=x$ whenever line 10 is reached.

Suppose we start the verification process with the candidate set comprising the boolean closure of the predicates $z=0, a=b$ and $y=x$. A possible counterexample is a path that passes through the first loop (lines 4-6) once, reaching line 7 with the abstract state $(a=$ $b \Rightarrow y \neq x) \wedge z \neq 0$, and then passes through the second loop (lines 7-9) once, reaching line 10 with the abstract

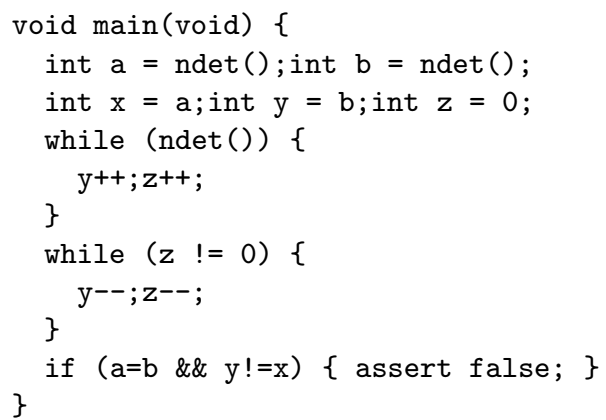

Fig. 1. A program on which CEGAR may diverge. state $z=0$, which does not imply $a=b \Rightarrow y=x$. A possible proof of the counterexample's spuriousness (i.e., proof that the path is actually safe) is the predicate $a=b \Rightarrow y=x+z$. The predicate turns out to be an inductive invariant for the program, and the verification process halts in the next iteration.

Unfortunately, the refinement process is not guaranteed to infer such a predicate but may choose any predicates that can prove the counterexample's safety. For instance, another possibility is the predicate $a=b \Rightarrow y=x+1$. Adding this to the candidate set is sufficient for proving the safety of the counterexample 
but not that of the program, and the abstraction process in the subsequent iteration would return yet another counterexample. For example, it may return the counterexample that passes the first loop twice, reaching line 7 with the abstract state $a=b \Rightarrow y \neq x+1$, and then passes through the second loop twice, reaching line 10 with the abstract state $z=0$ again. Then, the refinement process may choose the predicate $a=b \Rightarrow y=x+2$ to prove the spuriousness of this new counterexample, which is still insufficient to prove the whole program correct. The abstract-and-refine iteration may repeat indefinitely in this manner, adding to the candidates the predicate $a=b \Rightarrow y=x+i$ in each $i$-th run of the refinement process. A refinement process is said to be complete (w.r.t. $\mathcal{T}$ ) if the CEGAR process is guaranteed to converge and eventually discover a proof of the program's safety, when one exists in $\mathcal{T}$.

Previous works $[8,13]$ have proposed to achieve complete refinement in CEGAR by stratifying $\mathcal{T}$ into an infinite sequence of predicate languages $L^{0} \subseteq L^{1} \subseteq$ $\ldots L^{k} \ldots$ such that $\mathcal{T}=\bigcup_{k \in \omega} L^{k}$, and requiring each $i$-th run of the refinement to only infer predicates from the stratum $L^{\operatorname{lvl}(i)}$ where $l v l(i)$ is the stratum level at the $i$-th CEGAR iteration. By requiring each $L^{k}$ to be finite ${ }^{3}$ and raising the stratum level just when the refinement process reports that no proof exists for the given counterexample in the current stratum, the approach guarantees completeness. However, the approach requires the refinement process to exactly decide if there is a proof of the given counterexample in the current stratum. Indeed, completeness would be lost if the refinement process was allowed to report that the current stratum does not have a proof when it actually does. For many theories used in practice, such as the theory of linear real arithmetic, such an exact finite-language-restricted proof search incurs heavy overhead and is prohibitive (see Section 4, Section 5, and Appendix F for analysis and discussion).

The first contribution of this paper is the observation that exact finite language restricted proof search is actually unnecessary for completeness. Instead, we show that the following more relaxed scheme is sufficient: in each $i$-th run of the refinement process, we restrict the returned proof to some finite language of predicates $L_{\text {base }}^{\text {lvl }(i)} \cup L_{\text {ext }}^{\text {lvl }(i)}$ (base and extension) such that the refinement process may report that no proof exists only when no proof exists in $L_{b a s e}^{l v l(i)}$. There are no further restrictions on the refinement process, and so, the refinement process may return a proof that is not in $L_{\text {base }}$ (but in $L_{\text {base }} \cup L_{\text {ext }}$ ) even if a proof exists in $L_{\text {base }}$, or may report that no proof exists even if a proof exists in $L_{\text {base }} \cup L_{\text {ext }}$ (but not in $L_{\text {base }}$ ). We show that this relaxed approach still ensures completeness when the stratum level is raised just when the refinement process reports that there is no proof in the current stratum, as before, and $L_{\text {base }}$ grows to eventually cover $\mathcal{T}$ (i.e., $L_{\text {base }}^{0} \subseteq L_{\text {base }}^{1} \subseteq \ldots L_{\text {base }}^{k} \ldots$ such that $\left.\mathcal{T}=\bigcup_{k \in \omega} L_{\text {base }}^{k}\right)$. We formalize this observation in a refinement algorithm scheme called relaxed stratification (contra the exact stratification approach described above) and prove that it is indeed complete.

\footnotetext{
3 The term "finite predicate language" is used synonymously with "finite set of predicates".
} 
As the second contribution, we present a concrete refinement algorithm that implements the relaxed scheme. The algorithm is a modification of the proofbased refinement [6] in which the theory-level reasoning is restricted so that partial (tree-)interpolants at that level is restricted to $L_{\text {base }}{ }^{4}$ We also present a technique that uses a certain property of the counterexample patterns to further improve the efficiency of the algorithm while still satisfying the requirement of the scheme. We formalize the refinement algorithm as a constraint solver for recursion-free Horn-clause constraints $[4,14,15,24]$ which has gained popularity as the standard format for describing refinement algorithms. We have implemented a prototype of the refinement algorithm, and we show empirically that it is able to achieve complete predicate refinement with low overhead.

In summary, the paper's contributions are as follows:

- A new scheme for practical complete predicate refinement called relaxed stratification and the proof of its completeness (Section 2).

- A new predicate refinement algorithm as concrete instance of the relaxed stratification scheme (Section 3).

- Experiments with a prototype implementation of the refinement algorithm (Section 4).

The rest of the paper is organized as follows. Section 2 formally defines the relaxed stratification scheme and proves its completeness. Section 3 presents the concrete refinement algorithm implementing the scheme. Section 4 presents experimental results with the prototype implementation of the refinement algorithm. We discuss related work in Section 5 and conclude the paper in Section 6 . Appendix contains proofs and extra materials omitted from the main body of the paper.

\section{The Relaxed Stratification Scheme}

Let $\mathcal{T}$ be a FOL theory. For a formula $\theta$ in the signature of $\mathcal{T}$ (a $\mathcal{T}$-formula), we write $f v s(\theta)$ for the free variables in $\theta$. A predicate in $\mathcal{T}$ is of the form $\lambda x_{1}, \ldots, x_{n} . \theta$ where $\theta$ is a $\mathcal{T}$-formula such that $f v s(\theta) \subseteq\left\{x_{1}, \ldots, x_{n}\right\}$. For readability, we often omit the explicit $\lambda$ abstraction and treat a formula $\theta$ as the predicate $\lambda \bar{x} . \theta$ where $\{\bar{x}\}=f v s(\theta)$. We overload $\mathcal{T}$ for the set of predicates in $\mathcal{T}$.

\subsection{Assumptions on the Abstraction Process}

Relaxed stratification only concerns the refinement process part of CEGAR. We show that the scheme is quite general and can be used in a wide range of CEGAR-based verifiers. To this end, we delineate the conditions that the abstraction process part needs to satisfy. As we shall show below, the conditions are quite weak and satisfied by virtually any CEGAR-based verifier.

\footnotetext{
${ }^{4}$ While this approach has already been suggested in [8], they require the restricting language to be closed under conjunctions and disjunctions (see Section 5 for further discussion).
} 
We assume that the abstraction process Abs takes as input a program and a finite set of predicates in $\mathcal{T}$ (the set of candidate proofs). For a program $M$ and a finite set of predicates $F \subseteq \mathcal{T}$, we require that $\operatorname{Abs}(M, F)$ either returns safe, indicating that $M$ has been proved safe using the predicates from $F$, or returns a counterexample. For generality, we assume that a counterexample is also simply a program so that, for a counterexample $M$, we write $\operatorname{Abs}(M, F)=$ safe when $F$ is sufficient for the abstraction process to prove the spuriousness of $M$ (in practice, a counterexample is not an arbitrary program, but, e.g., an unwound program slice of the input program, and concrete instances of the relaxed stratification scheme take advantage of the counterexample structure - cf. Section 3). We sometimes say that $F$ refutes the counterexample $M$ when $\operatorname{Abs}(M, F)=$ safe.

We require Abs to be monotonic on the candidates, that is, if $\operatorname{Abs}(M, F)=$ safe and $F \subseteq F^{\prime}$ then $\operatorname{Abs}\left(M, F^{\prime}\right)=$ safe (i.e., having more predicates can only increase Abs's ability to prove). We also require that if $\operatorname{Abs}(M, F)=\operatorname{cex}\left(M^{\prime}\right)$ then $\operatorname{Abs}\left(M^{\prime}, F\right) \neq$ safe, that is, the returned counterexample is actually a counterexample and cannot be refuted by the given predicates. Finally, we require that if $\operatorname{Abs}(M, F)=$ safe and $\operatorname{Abs}\left(M, F^{\prime}\right)=\operatorname{cex}\left(M^{\prime}\right)$ then $\operatorname{Abs}\left(M^{\prime}, F\right)=$ safe, that is, if a set of predicates is a proof for program's safety then it is also a proof for any counterexample of the program. We say that Abs is sound when it only proves safe programs safe, that is, $\operatorname{Abs}(M, F)=$ safe only if $M$ is safe. ${ }^{5}$

We note that the assumptions on the abstraction process are quite liberal and do not demand, for example, the process uses the given set of predicates by decomposing them into atomic predicates and taking their boolean closure, taking the cartesian closure, or using them directly as loop invariants. In Example 1 below, we describe an example abstraction process that uses the predicates directly.

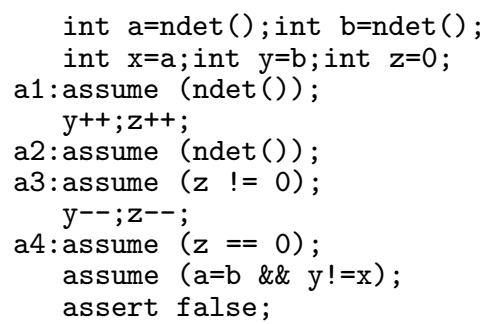

$$
M_{e x b}
$$

Fig. 2. Counterexamples of the program from Figure 1.

\footnotetext{
${ }^{5}$ Soundness of Abs is not required for completeness of relaxed stratification.
} 
Example 1. Let $\mathcal{T}$ be the quantifier-free theory of linear real arithmetic. Let Abs be the abstraction process that, given a program (or counterexample) $M$ and the set of predicates $F \subseteq \mathcal{T}$, checks if there exists an assignment from each loop-head location in $M$ to a predicate in $F$ that forms an inductive invariant of $M$. Recall the example from Section 1. Let $M_{e x}$ be the program shown in Figure 1. Then, the map $\rho$ such that $\rho(\mathrm{L} 4)=\rho(\mathrm{L} 7)=\theta_{e x 1}$ where $\theta_{e x 1} \equiv a=b \Rightarrow y=x+z$ is an inductive invariant of $M_{e x}$, and therefore, $\operatorname{Abs}\left(M_{e x},\left\{\theta_{e x 1}\right\}\right)=$ safe.

When given an insufficient set of predicates as the candidates, Abs returns a counterexample. For instance, as discussed in Section 1, a possible counterexample of $M_{e x}$ is $M_{e x a}$, shown in Figure 2, that passes through each loop once to reach line 10. (The semantics of assume $(b)$ is to safely halt if $b$ is false, and proceed otherwise.) Here, a1-a4 label the entry points of the unwound loops. Viewing them as one-iteration loops where invariants are asserted, it can be seen that $\rho$ such that $\rho(\ell)=\theta_{\text {ex1 }}$ for each $\ell \in\{\mathrm{a} 1-\mathrm{a} 4\}$ is an inductive invariant of $M_{\text {exa }}$, and therefore $\operatorname{Abs}\left(M_{\text {exa }},\left\{\theta_{\text {ex1 }}\right\}\right)=$ safe. However, as discussed in Section 1 , asserting $\theta_{0}$ at a1, a4, and $\theta_{1}$ at a2, a3 where $\theta_{0} \equiv a=b \Rightarrow y=x$ and $\theta_{1} \equiv a=b \Rightarrow y=x+1$ also constitutes a sufficient loop invariant of $M_{\text {exa }}$. Therefore, we also have $\operatorname{Abs}\left(M_{\text {exa }},\left\{\theta_{0}, \theta_{1}\right\}\right)=$ safe.

Similarly, $M_{e x b}$ shown in Figure 2 is a counterexample that passes through each loop twice to reach line 10 . By reasoning similar to the above, we have $\operatorname{Abs}\left(M_{e x b},\left\{\theta_{e x 1}\right\}\right)=\operatorname{Abs}\left(M_{e x b},\left\{\theta_{0}, \theta_{1}, \theta_{2}\right\}\right)=$ safe where $\theta_{2} \equiv a=b \Rightarrow y=x+2$.

\subsection{The Relaxed Stratification Scheme}

We are now ready to formalize the relaxed stratification scheme. The core of the scheme is the relaxed finite-language-restricted refinement process RIxRef that takes as input a counterexample and a restricting predicate language $\left(L_{\text {base }}, L_{\text {ext }}\right)$, and returns either unsafe indicating that the counterexample is real, a set of predicates $F \subseteq L_{\text {base }} \cup L_{e x t}$ that proves the safety of the counterexample, or noproof indicating that it could not find a proof for the counterexample within the given restriction.

We prepare strata of restricting predicate languages:

$$
\left(L_{\text {base }}^{0}, L_{\text {ext }}^{0}\right),\left(L_{\text {base }}^{1}, L_{\text {ext }}^{1}\right), \ldots\left(L_{\text {base }}^{k}, L_{\text {ext }}^{k}\right), \ldots
$$

We require each restricting predicate language to be finite, and the base-part to eventually cover $\mathcal{T}$. Formally, we impose the following condition on the restricting predicate languages: 1.) for each $k \in \omega, L_{b a s e}^{k} \cup L_{\text {ext }}^{k}$ is a finite subset of $\mathcal{T}$, 2.) for each $k \in \omega, L_{\text {base }}^{k} \subseteq L_{\text {base }}^{k+1}$, and 3.) $\mathcal{T}=\bigcup_{k \in \omega} L_{\text {base }}^{k}$.

Figure 3 shows the overview of the relaxed stratification verification process. The verification procedure RIxCegar takes as input the program $M$ to be verified, and first initializes the candidate predicate set Cands to $\varnothing$ (line 2) and the restricting language stratum $k$ to 0 (line 3). Then, it repeats the abstract-andrefine loop (lines 4-10) until convergence. The loop first calls $\operatorname{Abs}(M, C a n d s)$ to check if $M$ can be proved safe with the current candidates. If so, then we exit the verification process, returning safe (line 6). Otherwise, a counterexample 


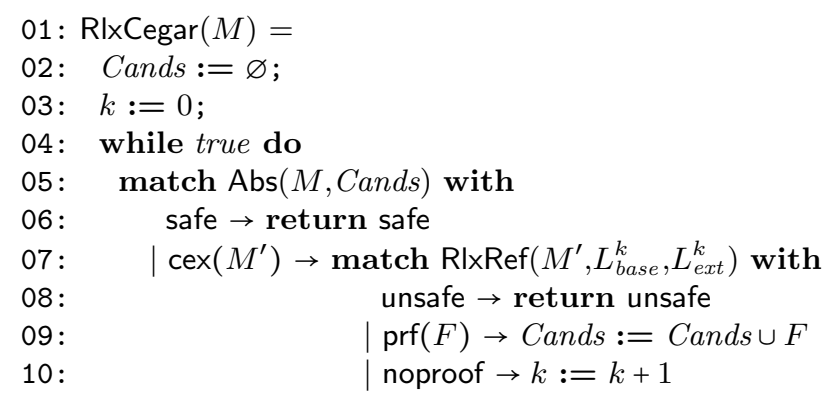

Fig. 3. The relaxed stratification verification process.

$M^{\prime}$ is obtained, and we call RlxRef on $M^{\prime}$ and the current restricting language $\left(L_{\text {base }}^{k}, L_{\text {ext }}^{k}\right.$ ) (line 7 ). If RlxRef returns unsafe, then the counterexample is real and we exit the verification process, returning unsafe (line 8). Otherwise, RIxRef either returns a set of predicates that refutes the counterexample (line 9), or returns noproof indicating that it has failed to find a proof for the counterexample in the current language stratum (line 10). In the former case, the returned set of predicates are added to Cands, and in the latter case, the language stratum is raised to the next level.

We require RIxRef to only report unsafe on a real counterexample, that is, $\operatorname{RIx} \operatorname{Ref}\left(M, L_{\text {base }}, L_{\text {ext }}\right)=$ unsafe only if $\forall F \subseteq \mathcal{T} \cdot \operatorname{Abs}(M, F) \neq$ safe, and we require the returned proof to be actually a proof of the counterexample, that is $\operatorname{RIxRef}\left(M, L_{\text {base }}, L_{\text {ext }}\right)=\operatorname{prf}(F)$ only if $\operatorname{Abs}(M, F)=$ safe (these conditions are not particular to relaxed stratification and usually assumed for any refinement process in CEGAR). In addition, we require RIxRef to only infer proofs from the given restricting predicate language and be able to return some proof if the given counterexample is refutable just in the base part of the language. Formally, we impose the following additional conditions on RIxRef:

- If $\operatorname{RIxRef}\left(M, L_{\text {base }}, L_{\text {ext }}\right)=\operatorname{prf}(F)$ then $F \subseteq L_{\text {base }} \cup L_{\text {ext }}$; and

- If $\exists F \subseteq L_{\text {base }} \cdot \operatorname{Abs}(M, F)=$ safe, then $\operatorname{RlxRef}\left(M, L_{\text {base }}, L_{\text {ext }}\right)=\operatorname{prf}\left(F^{\prime}\right)$ for some $F^{\prime}$.

We state and prove the completeness of the relaxed stratification scheme.

Theorem 1 (Completeness). If $\exists F \subseteq \mathcal{T}$.Abs $(M, F)=$ safe, then RlxCegar $(M)$ terminates and returns safe.

We remind that safety verification is undecidable in general, and our "completeness" only states that the verification terminates under the promise that a proof of the program's safety exists in $\mathcal{T}{ }^{6}$

Also, assuming that Abs is sound (cf. Section 2.1), it is easy to see that $\mathrm{RIxCegar}$ is also sound in that it only proves safe programs safe (in fact, this holds independently of the behavior of RIxRef).

\footnotetext{
${ }^{6}$ This notion of completeness is the same as the one from previous works $[8,13]$.
} 
Theorem 2 (Soundness). If Abs is sound, then RlxCegar( $M)$ returns safe only if $M$ is safe.

Example 2. We show how the relaxed stratification scheme would ensure the convergence of a verifier on the program $M_{e x}$ from Example 1. Suppose that we run RIxCegar $\left(M_{e x}\right)$, and for contradiction, it diverges by generating the following infinite series of refinements discussed in Section 1:

$$
a=b \Rightarrow y=x, \quad a=b \Rightarrow y=x+1, \quad \ldots \quad a=b \Rightarrow y=x+i, \quad \ldots
$$

By the definition of RlxCegar, it must be the case that the restricting predicate language at the $i$-th CEGAR iteration is $\left(L_{\text {base }}^{\text {lvl }(i)}, L_{\text {ext }}^{\text {lvl }(i)}\right)$ such that $y=x+i \epsilon$ $L_{\text {base }}^{l v l(i)} \cup L_{\text {ext }}^{l v l(i)}$ where $l v l(i)$ is the restricting language stratum level in the $i$-th iteration. Because each $L_{b a s e}^{l v l(i)} \cup L_{e x t}^{l v l(i)}$ is finite, the stratum level of the language must have been raised infinitely many times. Therefore, $a=b \Rightarrow y=x+z \in L_{\text {base }}^{j}$ for some $j$ because $\mathcal{T}=\bigcup_{k \in \omega} L_{\text {base }}^{k}$.

But, as argued in Example 1, $a=b \Rightarrow y=x+z$ is a sufficient proof of $M_{e x}$ 's safety, and therefore also that of its counterexamples. Therefore, by the fact that RIxRef refutes any counterexample refutable in the base part of the given restricting language, for any counterexample $M^{\prime}$ of $M_{e x}, \operatorname{RlxRef}\left(M^{\prime}, L_{\text {base }}^{j}, L_{\text {ext }}^{j}\right)=$ $\operatorname{prf}\left(F^{\prime}\right)$ for some $F^{\prime} \subseteq L_{\text {base }}^{j} \cup L_{\text {ext }}^{j}$. Then, because $L_{\text {base }}^{j} \cup L_{\text {ext }}^{j}$ is finite, RlxCegar must have eventually inferred a sufficient set of predicates that constitutes a proof of $M_{e x}$ 's safety without further raising the language stratum.

\section{Concrete Refinement Algorithm Instances}

We show how to implement the relaxed finite-language-restricted refinement process RIxRef. In fact, we describe a technique that takes as module an exact $L_{\text {base }}$-restricted refinement algorithm and turn it into a relaxed $\left(L_{\text {base }}, L_{\text {base }}{ }^{\wedge \vee}\right)$ restricted refinement algorithm. (We write $L^{\wedge \vee}$ for the closure of $L$ under conjunctions and disjunctions.) We focus on the case where the given counterexample is spurious. ${ }^{7}$

Following the recent trend $[20,4,10,3,2,15,14,24]$, we formalize the refinement algorithm as a constraint solver for recursion-free Horn-clause constraints. Specifically, we present a relaxed $\left(L_{\text {base }}, L_{\text {ext }}\right)$-restricted constraint solver that takes as module an exact $L_{\text {base }}$-restricted constraint solver (cf. Section 3.1 for the definition of exact/relaxed finite-language-restricted constraint solvers). We review Horn-clause constraints in Section 3.1, and describe the constraint solver, that we call RIxSolveA, in Section 3.2.

We also present a technique that takes as module a relaxed $\left(L_{\text {base }}, L_{\text {ext }}\right)$ restricted constraint solver, an unrestricted constraint solver $\mathcal{A U}$, and a positive integer parameter $\ell$, and turn them into a relaxed $\left(L_{\text {base }}, \operatorname{LB}\left(L_{\text {base }} \cup L_{\text {ext }}, \mathcal{A U}, \ell\right)\right)$ restricted constraint solver where $\operatorname{LB}(L, \mathcal{A U}, \ell)$ is a certain finite language of

\footnotetext{
${ }^{7}$ Detecting if the counterexample real and returning unsafe if so can be handled via usual unrestricted refinement (cf. Section 4).
} 
predicates determined by $L, \mathcal{A U}$, and $\ell$. We formalize the technique as the constraint solver RlxSolveB, described in Section 3.3. The technique applies the relaxed finite-language-restricted constraint solver provided as the module to only a small subset of the constraint solving problem, and can be used to improve the efficiency of the given relaxed finite-language-restricted constraint solver.

We remind that the exact finite-language-restricted proof search is an inherently expensive process (cf. Section 5, Section 4, and Appendix F), and the key idea in these constraint solvers is to use the expensive exact finite-languagerestricted proof search process (given as a module) only on small subparts of the problem. This is made possible thanks to the relaxed requirement on the language restriction where the refinement process is not required to exactly decide the existence of a restricted solution for the whole problem. Informally, the trick is to choose the subproblems just large enough to guarantee that if a subproblem is not $L_{\text {base }}$ solvable then neither is the whole and that there can only be finitely many solutions for the whole obtainable from $L_{b a s e}$-restricted solutions for the subproblems.

\subsection{Horn Clause Constraints}

For concreteness, in what follows, we assume that the underlying theory $\mathcal{T}$ is the quantifier-free theory of linear real arithmetic (QFLRA). However, the techniques presented in Sections 3.2 and 3.3 can be applied to any quantifier-free theory.

A formula $\theta$ in the signature of QFLRA comprises atomic predicate $p$ of the form $a_{1} x_{1}+a_{2} x_{2} \cdots+a_{n} x_{n} \leq a_{n+1}$ where $a_{1}, \ldots, a_{n+1} \in \mathbb{Z}$, and is closed under the usual boolean operations $\neg, \wedge, \vee$, and $\Rightarrow$. As usual, we let $\neg$ bind the tightest and $\Rightarrow$ the weakest. A literal $l$ is either an atomic predicate or its negation. A clause $C$ is a disjunction of literals. A conjunctive normal form (CNF) is a conjunction of clauses. We often use a set to represent a clause or a CNF so that $\left\{l_{1}, \ldots, l_{n}\right\}$ represents $l_{1} \vee \cdots \vee l_{n}$ and $\left\{C_{1}, \ldots, C_{n}\right\}$ represents $C_{1} \wedge \cdots \wedge C_{n}$. We write $\perp$ for contradiction and $\mathrm{T}$ for tautology. We write $\vDash \theta$ when $\theta$ is valid in $\mathcal{T}$.

Horn Clauses and Horn-Clause Constraints. A predicate variable application is of the form $P(\bar{x})$ where $P$ is a predicate variable of arity $|\bar{x}|$. A Horn clause $h c$ is of the form $\theta \wedge B_{1} \wedge \cdots \wedge B_{n} \rightarrow H$ where $\theta$ is a formula in $\mathcal{T}$, each $B_{i}$ is a predicate variable application, and $H$ is a predicate variable application or $\perp$. We call $H$ the head of the Horn clause, and $\theta \wedge B_{1} \wedge \cdots \wedge B_{n}$ the body. A Horn clause whose head is $\perp$ is called a root clause.

A Horn-clause constraint set (HCCS) $\mathcal{H}$ is a finite set of Horn clauses. We write $\operatorname{pvs}(\mathcal{H})$ for the predicate variables in $\mathcal{H}$. We write leaves $(\mathcal{H})$ for the set of predicate variables in $\mathcal{H}$ that do not occur as a head in $\mathcal{H}$. We define $\sim \mathcal{H}$ to be the relation $\{(P, Q) \mid \theta \wedge \ldots P(\bar{x}) \ldots \rightarrow Q(\bar{y}) \in \mathcal{H}\}$. We say that a Horn clause $\theta \wedge B_{1} \wedge \cdots \wedge B_{n} \rightarrow H$ is conjunctive if $\theta$ is a conjunction of literals. We say that an HCCS $\mathcal{H}$ is conjunctive if each $h c \in \mathcal{H}$ is conjunctive.

We say that $\mathcal{H}$ is recursion-free if $\sim \mathcal{H}$ is acyclic. We say that a recursion-free HCCS $\mathcal{H}$ is tree-like [14,15] if 1.) there is exactly one root clause in $\mathcal{H}$ and every 


$$
\begin{aligned}
& \theta_{\mathrm{p} 1} \rightarrow P(\bar{x}) \quad \theta_{\mathrm{p} 1} \rightarrow P_{1}(\bar{x}) \quad \theta_{\mathrm{p} 1} \rightarrow P_{1}(\bar{x}) \\
& \theta_{\mathrm{p} 2} \wedge P(\bar{x}) \rightarrow P\left(\bar{x}^{\prime}\right) \quad \theta_{\mathrm{p} 2} \wedge P_{1}(\bar{x}) \rightarrow P_{2}\left(\bar{x}^{\prime}\right) \quad \theta_{\mathrm{p} 2} \wedge P_{1}(\bar{x}) \rightarrow P_{2}\left(\bar{x}^{\prime}\right)
\end{aligned}
$$

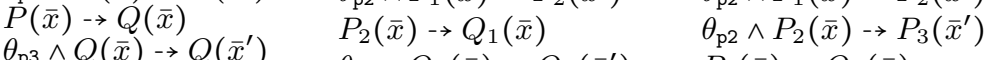

$$
\begin{aligned}
& \begin{array}{ll}
\theta_{\mathrm{p} 3} \wedge Q(\bar{x}) \rightarrow Q\left(\bar{x}^{\prime}\right) & \theta_{\mathrm{p} 3} \wedge Q_{1}(\bar{x}) \rightarrow Q_{2}\left(\bar{x}^{\prime}\right) \quad P_{3}(\bar{x}) \rightarrow Q_{1}(\bar{x}) \\
\theta_{\mathrm{p} 4} \wedge Q(\bar{x}) \rightarrow \perp & \theta_{\mathrm{p} 4} \wedge Q_{2}(\bar{x}) \rightarrow \perp
\end{array} \\
& \theta_{\mathrm{p} 4} \wedge Q(\bar{x}) \rightarrow 1 \quad \theta_{\mathrm{p} 4} \wedge Q_{2}(\bar{x}) \rightarrow \perp \quad \theta_{\mathrm{p} 3} \wedge Q_{1}(\bar{x}) \rightarrow Q_{2}\left(\bar{x}^{\prime}\right) \\
& \theta_{\mathrm{p} 3} \wedge Q_{2}(\bar{x}) \rightarrow Q_{3}\left(\bar{x}^{\prime}\right) \\
& \mathcal{H}_{\text {ex }} \mathcal{H}_{\text {exa }} \quad \mathcal{H}_{\text {exb }}
\end{aligned}
$$

Fig. 4. HCCS examples.

$P \in \operatorname{pvs}(\mathcal{H})$ can reach a predicate variable occurring in the body of the root clause via $\sim_{\mathcal{H}}^{*}$; and 2.) for any $P \in \operatorname{pvs}(\mathcal{H})$, at most one $h c \in \mathcal{H}$ contains $P$ in its body, at most one $h c \in \mathcal{H}$ contains $P$ as its head, and no $h c \in \mathcal{H}$ has multiple occurrences of $P$. For a tree-like HCCS $\mathcal{H}$, we define the depth of $\mathcal{H}$, depth $(\mathcal{H})$, to be the length of the longest $\sim \mathcal{H}$ path. For $\eta$ a mapping from predicate variables to predicate variables, we write $\eta(h c)$ for the Horn clause $h c$ with each predicate variable application $P(\bar{x})$ replaced by $\eta(P)(\bar{x})$. We write $\eta(\mathcal{H})$ for $\{\eta(h c) \mid h c \in \mathcal{H}\}$. We say that a tree-like HCCS $\mathcal{H}^{\prime}$ is an unwound instance of a (possibly recursive) HCCS $\mathcal{H}$ if there exists a mapping $\eta$ from $p v s\left(\mathcal{H}^{\prime}\right)$ to $p v s(\mathcal{H})$ such that $\eta\left(\mathcal{H}^{\prime}\right) \subseteq \mathcal{H}$.

Constraint Solutions and Restricted Constraint Solvers. For $\sigma$ a mapping from predicate variables to predicates in $\mathcal{T}$, we write $\sigma(h c)$ for $h c$ with each predicate variable application $P(\bar{x})$ replaced by $\theta[\bar{x} / \bar{y}]$ where $\sigma(P)=\lambda \bar{y}$. $\theta$. We say that the map $\sigma$ from $\operatorname{pvs}(\mathcal{H})$ to predicates in $\mathcal{T}$ is a solution of $\mathcal{H}$, written $\sigma \vDash \mathcal{H}$, if for each $h c \in \mathcal{H}, \vDash \sigma(h c)$, interpreting $\rightarrow$ as $\Rightarrow$. We define $\operatorname{ran}(\sigma)$, the range of $\sigma$, to be the set of predicates $\{\sigma(P) \mid P \in \operatorname{dom}(\sigma)\}$.

We focus on constraint solving algorithms for tree-like HCCSs (they can be extended to arbitrary recursion-free HCCSs by adopting the technique from [15]). We say that an algorithm is an unrestricted constraint solver if given a tree-like HCCS $\mathcal{H}$, it returns a solution of $\mathcal{H}$ or decides that $\mathcal{H}$ has no solution. We say that an algorithm is an exact L-restricted constraint solver if given a tree-like HCCS $\mathcal{H}$, it decides if there is a solution $\sigma$ of $\mathcal{H}$ such that $\operatorname{ran}(\sigma) \subseteq L$ and returns such a solution if so. We say that an algorithm is a relaxed $\left(L_{\text {base }}, L_{\text {ext }}\right)$-restricted constraint solver if given a tree-like $\operatorname{HCCS} \mathcal{H}$, it either returns a solution $\sigma$ of $\mathcal{H}$ such that $\operatorname{ran}(\sigma) \subseteq L_{\text {base }} \cup L_{\text {ext }}$ or returns noproof indicating that it has failed to find a solution, with the requirement that it returns some solution (whose range is in $\left.L_{\text {base }} \cup L_{\text {ext }}\right)$ if there exists a solution $\sigma^{\prime}$ of $\mathcal{H}$ such that $\operatorname{ran}\left(\sigma^{\prime}\right) \subseteq L_{\text {base }}$.

Example 3. Consider the HCCS $\mathcal{H}_{e x}$ shown in Figure 4. Here, $\bar{x}=a, b, x, y, z$, $\bar{x}^{\prime}=a^{\prime}, b^{\prime}, x^{\prime}, y^{\prime}, z^{\prime}$, and

$$
\begin{aligned}
\theta_{\mathrm{p} 1} & \equiv x=a \wedge y=b \wedge z=0 \\
\theta_{\mathrm{p} 2} & \equiv z^{\prime}=z+1 \wedge y^{\prime}=y+1 \wedge x^{\prime}=x \wedge a^{\prime}=a \wedge b^{\prime}=b \\
\theta_{\mathrm{p} 3} & \equiv z \neq 0 \wedge z^{\prime}=z-1 \wedge y^{\prime}=y-1 \wedge x^{\prime}=x \wedge a^{\prime}=a \wedge b^{\prime}=b \\
\theta_{\mathrm{p} 4} & \equiv z=0 \wedge a=b \wedge x \neq y
\end{aligned}
$$


$\mathcal{H}_{e x}$ is not tree-like (in fact, $\sim_{S}$ is cyclic). Figure 4 shows HCCSs $\mathcal{H}_{\text {exa }}$ and $\mathcal{H}_{\text {exb }}$ that are tree-like. In addition, they are unwound instances of $\mathcal{H}_{e x}$ because $\eta_{a}\left(\mathcal{H}_{e x a}\right) \subseteq \mathcal{H}_{e x}$ and $\eta_{b}\left(\mathcal{H}_{e x b}\right) \subseteq \mathcal{H}_{e x}$ where $\eta_{a}=\left\{P_{1} \mapsto P, P_{2} \mapsto P, Q_{1} \mapsto Q, Q_{2} \mapsto Q\right\}$ and $\eta_{b}=\left\{P_{1} \mapsto P, P_{2} \mapsto P, P_{3} \mapsto P, Q_{1} \mapsto Q, Q_{2} \mapsto Q, Q_{3} \mapsto Q\right\}$.

Recall the predicates $\theta_{\text {ext1 }}, \theta_{0}, \theta_{1}, \theta_{2}$ from Example 1. Let the maps $\sigma_{a_{1}}, \sigma_{a_{2}}$, $\sigma_{b_{1}}$, and $\sigma_{b_{2}}$ be defined as below.

$$
\begin{aligned}
& \sigma_{a_{1}}=\left\{P \mapsto \theta_{\text {ex1 }} \mid P \in \operatorname{pvs}\left(\mathcal{H}_{e x a}\right)\right\} \quad \sigma_{b_{1}}=\left\{P \mapsto \theta_{\text {ex1 }} \mid P \in \operatorname{pvs}\left(\mathcal{H}_{e x b}\right)\right\} \\
& \sigma_{a_{2}}=\left\{P \mapsto \theta_{0} \mid P \in\left\{P_{1}, Q_{2}\right\}\right\} \quad \sigma_{b_{2}}=\left\{P \mapsto \theta_{0} \mid P \in\left\{P_{1}, Q_{3}\right\}\right\} \\
& \cup\left\{P \mapsto \theta_{1} \mid P \in\left\{P_{2}, Q_{1}\right\}\right\} \quad \cup\left\{P \mapsto \theta_{1} \mid P \in\left\{P_{2}, Q_{2}\right\}\right\} \\
& \cup\left\{P \mapsto \theta_{2} \mid P \in\left\{P_{3}, Q_{1}\right\}\right\}
\end{aligned}
$$

Then, $\sigma_{a_{1}}$ and $\sigma_{a_{2}}$ are solutions of $\mathcal{H}_{\text {exa }}$, and $\sigma_{b_{1}}$ and $\sigma_{b_{2}}$ are solutions of $\mathcal{H}_{\text {exb }}$.

Relating Refinement Process to Constraint Solving. We relate constraint solving to refinement process. Roughly, the relationship says that, for any counterexample, there is a corresponding tree-like HCCS such that the range of its solutions are the proofs of the counterexample's spuriousness. We further assume that such a tree-like HCCS is always an unwound instance of some fixed "generator" HCCS determined by the given program.

We formalize the relationship. Let $M$ be a program. We assume that there exists an HCCS $\mathcal{H}_{g e n(M)}$ such that for any counterexample $M^{\prime}$ of $M$ (i.e., $\operatorname{Abs}(M, F)=\operatorname{cex}\left(M^{\prime}\right)$ for some $\left.F\right)$, there exists an unwound instance $\mathcal{H}_{M^{\prime}}$ of $\mathcal{H}_{\text {gen }(M)}$ that satisfies the following:

- if $\sigma \vDash \mathcal{H}_{M^{\prime}}$ then $\operatorname{Abs}\left(M^{\prime}, \operatorname{ran}(\sigma)\right)=$ safe (i.e., the range of a solution of $\mathcal{H}_{M^{\prime}}$ is a proof of $M^{\prime}$ 's spuriousness); and

- if $\operatorname{Abs}\left(M^{\prime}, F\right)=$ safe then $\exists \sigma \cdot \operatorname{ran}(\sigma) \subseteq F \wedge \sigma \vDash \mathcal{H}_{M^{\prime}}$ (i.e., if $M^{\prime}$ can be refuted by $F$, then there is a solution for $\mathcal{H}_{M^{\prime}}$ whose range is in $F$ ).

Hence, the task of implementing a relaxed language restricted refinement process RIxRef for the restricting language $\left(L_{\text {base }}, L_{\text {ext }}\right)$ is now reduced to implementing a relaxed $\left(L_{\text {base }}, L_{\text {ext }}\right)$-restricted constraint solver.

We remark that the relationship stated above is quite general and many CEGAR-based verifiers $[20,10,3,2,15,14,24]$ use the relationship to implement the refinement process as a constraint solver for tree-like HCCSs. For example, refuting a counterexample in a typical CEGAR-based verification of sequential imperative programs is equivalent to solving a tree-like HCCS of the form below where $\bar{x}$ are the variables in the program, and each $\theta_{i}$ is a formula on $\bar{x}$ and $\bar{x}^{\prime}$ that expresses the semantics of symbolically executing the corresponding segment (e.g., basic block) in the path:

$$
\begin{aligned}
& \begin{array}{lc}
\theta_{1} \rightarrow P_{1}(\bar{x}) & \theta_{i} \wedge P_{i}(\bar{x}) \rightarrow P_{i+1}\left(\bar{x}^{\prime}\right) \\
\theta_{2} \wedge P_{1}(\bar{x}) \rightarrow P_{2}\left(\bar{x}^{\prime}\right) & \vdots
\end{array}
\end{aligned}
$$

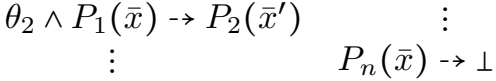


In such a verification, the generator HCCS $\mathcal{H}_{g e n(M)}$ can be described as follows. Let $\bar{x}$ be the variables in the program. For each node $a$ in the program's control flow graph $(\mathrm{CFG})$, we associate a predicate variable $P_{a}$ of arity $|\bar{x}|$. For each edge from node $a$ to node $b$ in the CFG, we add to $\mathcal{H}_{g e n(M)}$ the Horn clause $\theta_{a b} \wedge P_{a}(\bar{x}) \rightarrow P_{b}\left(\bar{x}^{\prime}\right)$ where $\theta_{a b}$ is a formula on $\bar{x}$ and $\bar{x}^{\prime}$ expressing the effect of symbolically executing the CFG path from $a$ to $b$ (with $\bar{x}$ representing the current and $\bar{x}^{\prime}$ representing the post state). For the entry node $a$, we add the Horn clause $\theta_{\text {init }} \rightarrow P_{a}(\bar{x})$ where $\theta_{\text {init }}$ is a formula on $\bar{x}$ expressing the program's initial state. Finally, for each error node $a$ (i.e., assert false statement), we add the Horn clause $P_{a}(\bar{x}) \rightarrow \perp$.

Example 4. Recall the program $M_{e x}$ from Example 1. The corresponding generator HCCS $\mathcal{H}_{g e n\left(M_{e x}\right)}$ is $\mathcal{H}_{e x}$ from Example 3. Roughly, the predicate variable $P$ in the HCCS represents the program states at the time when the first loop is entered, and $Q$ represents the states when the second loop is entered.

Recall the counterexamples $M_{e x a}$ and $M_{e x b}$ from Example 1, and the treelike HCCSs $\mathcal{H}_{\text {exa }}$ and $\mathcal{H}_{\text {exb }}$ from Example 3. $\mathcal{H}_{\text {exa }}$ corresponds to $M_{\text {exa }}$ and $\mathcal{H}_{\text {exb }}$ corresponds to $M_{\text {exb }}$. Indeed, as shown in Example 1, $\left\{\theta_{\text {ext }}\right\}$ (resp. $\left\{\theta_{0}, \theta_{1}\right\}$ ) is a proof of $M_{e x a}$, and $\mathcal{H}_{e x a}$ has the corresponding solution $\sigma_{a_{1}}$ (resp. $\sigma_{a_{2}}$ ) from Example 3. Similarly, the solutions $\sigma_{b_{1}}$ and $\sigma_{b_{2}}$ of $\mathcal{H}_{e x b}$ and the proofs $\left\{\theta_{e x 1}\right\}$ and $\left\{\theta_{0}, \theta_{1}, \theta_{2}\right\}$ of $M_{e x b}$ correspond.

\subsection{The Constraint Solver RIxSolveA}

RlxSolveA is a relaxed $\left(L_{\text {base }}, L_{\text {base }}{ }^{\wedge \vee}\right)$-restricted constraint solver. It is parameterized by an exact $L_{\text {base-restricted constraint solver that it takes as module. }}$

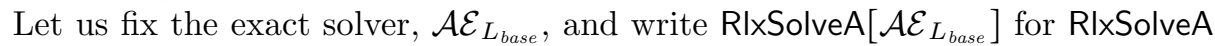
parameterized by the exact solver. Note that $L_{\text {base }}{ }^{\wedge}$ is finite for a finite $L_{\text {base }}$.

We briefly overview the construction of RIxSolveA. First, we leverage the equivalence of solving tree-like HCCS and tree interpolation [15] to reduce the problem to tree interpolation. Then, we adopt the standard proof-based interpolation technique that obtains interpolants from resolution proofs generated via SMT solving [12], except that we modify the SMT solver to use the exact $L_{\text {base-restricted solver }} \mathcal{A E}_{L_{\text {base }}}$ for the theory solver so as to infer $L_{\text {base-restricted }}$ (partial) interpolants at the theory level of the resolution proof. As we shall show, this guarantees that if the SMT solver fails to prove, then no $L_{\text {base }}$ solution exists, and conversely, any inferred solution is guaranteed to be in $L_{\text {base }} \wedge \vee$.

We describe the approach more formally. First, we review tree interpolation. The tree interpolation problem takes as input $(V, E, \Theta)$ where $(V, E)$ is a finite directed tree with the node set $V$ and $\left(v, v^{\prime}\right) \in E$ denoting that the node $v$ is a direct child of the node $v^{\prime}$, and the map $\Theta$ labels each node $v \in V$ with the $\mathcal{T}$-formula $\Theta(v)$. The goal is to find a map $I$ from $V$ to $\mathcal{T}$-formulas, called a tree interpolant of $(V, E, \Theta)$, that satisfies the following.

- $I\left(v_{r t}\right)=\perp$ for the root node $v_{r t}$

- for each $v \in V, \vDash \Theta(v) \wedge \wedge_{\left(v^{\prime}, v\right) \in E} I\left(v^{\prime}\right) \Rightarrow I(v)$; and 
- for each $v \in V, f v s(I(v)) \subseteq\left(\bigcup_{\left(v^{\prime}, v\right) \in E^{*}} f v s\left(\Theta\left(v^{\prime}\right)\right)\right) \cap\left(\bigcup_{\left(v^{\prime}, v\right) \notin E^{*}} f v s\left(\Theta\left(v^{\prime}\right)\right)\right)$.

We reduce constraint solving for a tree-like HCCS to tree interpolation as follows. ${ }^{8}$ Let $\mathcal{H}$ be the input tree-like HCCS. We transform $\mathcal{H}$ to an equivalent HCCS that satisfies: 1.) for each predicate variable $P \in p v s(\mathcal{H})$, there exists a vector of fresh variables $\bar{x}_{P}$ such that $P$ only occurs in the form $P\left(\bar{x}_{P}\right)$, and 2.) the only sharing of variables among Horn clauses are $\bar{x}_{P}$ 's between two Horn clauses both containing $P$. Then, the transformed $\mathcal{H}$ is reduced to the tree interpolation problem $\left(V_{\mathcal{H}}, E_{\mathcal{H}}, \Theta_{\mathcal{H}}\right)$ where

$-V_{\mathcal{H}}=\operatorname{pvs}(\mathcal{H}) \cup\left\{v_{r t}\right\}$ where $v_{r t} \notin p v s(\mathcal{H}) ;$

$-E_{\mathcal{H}}=\sim_{\mathcal{H}} \cup\left\{\left(P, v_{r t}\right) \mid \theta \wedge \ldots P\left(\bar{x}_{P}\right) \ldots \rightarrow \perp \in \mathcal{H}\right\}$

- For each $P, \Theta_{\mathcal{H}}(P)=\theta_{P}$ if $\theta_{P} \wedge \bigwedge_{i} B_{i} \rightarrow P\left(\bar{x}_{P}\right) \in \mathcal{H}$ and otherwise $\Theta_{\mathcal{H}}(P)=\perp$; and

- $\Theta_{\mathcal{H}}\left(v_{r t}\right)=\theta_{r t}$ where $\theta_{r t} \wedge \wedge_{i} B_{i} \rightarrow \perp \in \mathcal{H}$.

The theorem below follows from the construction, and shows the one-to-one correspondence between the tree interpolants of $\left(V_{\mathcal{H}}, E_{\mathcal{H}}, \Theta_{\mathcal{H}}\right)$ and the solutions of $\mathcal{H}$.

Theorem 3 ([15]). Let $\mathcal{H}$ be a tree-like HCCS. Let $\sigma$ and $I$ be such that $I\left(v_{r t}\right)=$ $\perp$ and for each $P \in \operatorname{pvs}(S), \sigma(P)=\lambda \bar{x}_{P} . I(P)$. Then, $\sigma \vDash \mathcal{H}$ if and only if $I$ is a tree interpolant of $\left(V_{\mathcal{H}}, E_{\mathcal{H}}, \Theta_{\mathcal{H}}\right)$.

Example 5. Recall the tree-like HCCS $\mathcal{H}_{\text {exa }}$ from Example 3. The corresponding tree interpolation problem $(V, E, \Theta)$ is shown below where each $\bar{x}_{P_{1}}, \bar{x}_{P_{2}}, \bar{x}_{Q_{1}}$, $\bar{x}_{Q_{2}}$ is a quintuple of fresh variables.

$$
\begin{aligned}
& V=\left\{v_{r t}, P_{1}, P_{2}, Q_{1}, Q_{2}\right\} \\
& E=\left\{\left(P_{1}, P_{2}\right),\left(P_{2}, Q_{1}\right),\left(Q_{1}, Q_{2}\right),\left(Q_{2}, v_{r t}\right)\right\} \\
& \Theta\left(P_{1}\right)=\theta_{\mathrm{p} 1}\left[\bar{x}_{P_{1}} / \bar{x}\right] \quad \Theta\left(P_{2}\right)=\theta_{\mathrm{p} 2}\left[\bar{x}_{P_{1}} / \bar{x}\right]\left[\bar{x}_{P_{2}} / \bar{x}^{\prime}\right] \\
& \Theta\left(Q_{1}\right)=\bar{x}_{P_{2}}=\bar{x}_{Q_{1}} \quad \Theta\left(Q_{2}\right)=\theta_{\mathrm{p} 3}\left[\bar{x}_{Q_{1}} / \bar{x}\right]\left[\bar{x}_{Q_{2}} / \bar{x}^{\prime}\right] \\
& \Theta\left(v_{r t}\right)=\theta_{\mathrm{p} 4}\left[\bar{x}_{Q_{2}} / \bar{x}\right]
\end{aligned}
$$

Now, the relaxed ( $L_{\text {base }}, L_{\text {base }}{ }^{\wedge}$ ) -restricted constraint solving problem is reduced to relaxed ( $L_{\text {base }}, L_{\text {base }}{ }^{\wedge \vee}$ )-restricted tree interpolation. That is, we would like to find tree interpolants restricted to $L_{\text {base }} \cup L_{\text {base }}{ }^{\wedge}$ (i.e., $L_{\text {base }}{ }^{\wedge \vee}$ ), with the guarantee to return one if there exists a $L_{\text {base }}$-restricted tree interpolant.

Next, we describe the process of relaxed $\left(L_{\text {base }}, L_{\text {base }}{ }^{\wedge \vee}\right)$-restricted tree interpolation. In what follows, we assume familiarity with lazy SMT and the proofbased technique for obtaining interpolants from resolution proofs [19,12]. Let $(V, E, \Theta)$ be the tree interpolation instance to be solved. In an ordinary proofbased tree interpolation, one looks for tree interpolants by having the SMT solver check the unsatisfiability of $\wedge_{v \in V} \Theta(v)$ and analyzing the output resolution proof to compute the interpolant. However, this decides the existence of, and infers,

\footnotetext{
8 The reduction is adopted from [15].
} 


$$
\begin{aligned}
& \text { THY } \frac{\mathcal{A E}_{L_{\text {base }}}\left(\mathcal{H}_{\text {thy }(C, V, E, \Theta)}\right)=\sigma \quad I\left(v_{r t}\right)=\perp \quad \forall P \cdot \lambda \bar{x}_{P} \cdot I(P)=\sigma(P)}{(V, E, \Theta) \vdash_{i t p} C: I} \\
& C \in \Theta(v) \quad \forall v^{\prime} . I\left(v^{\prime}\right)= \begin{cases}C \uparrow_{v^{\prime}} & \text { if }\left(v, v^{\prime}\right) \in E^{*} \\
\top & \text { otherwise }\end{cases} \\
& \text { HYP }(V, E, \Theta) \vdash_{i t p} C: I \\
& \begin{array}{l}
(V, E, \Theta) \vdash_{i t p} p \vee C_{1}: I_{1} \\
(V, E, \Theta) \vdash_{i t p} \neg p \vee C_{2}: I_{2}
\end{array} \quad \forall v \cdot I_{3}(v)= \begin{cases}I_{1}(v) \wedge I_{2}(v) & \text { if } p \in \text { outs }(v) \\
I_{1}(v) \vee I_{2}(v) & \text { otherwise }\end{cases} \\
& \operatorname{RES}(V, E, \Theta) \vdash_{i t p} C_{1} \vee C_{2}: I_{3}
\end{aligned}
$$

Fig. 5. The tree interpolation rules.

a tree interpolant from the entire $\mathcal{T}$, and is unsuitable for our task (i.e., this results in an unrestricted constraint solver).

Instead, we modify the SMT solver so that its theory-level reasoning is del-

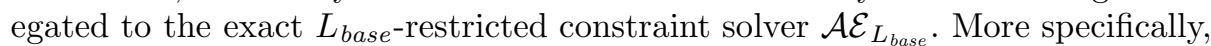
when the SMT solver builds a model of possible (propositional) satisfying assignment $\neg C$, instead of passing the model to a theory solver as in ordinary SMT, we build a "fragment" HCCS $\mathcal{H}_{t h y(C, V, E, \Theta)}$ that just contains the part of the tree interpolation problem touched by the literals in $C$. Formally,

$$
\mathcal{H}_{t h y(C, V, E, \Theta)}=\left\{\neg C \downarrow_{v} \wedge \wedge_{(P, v) \in E} P\left(\bar{x}_{P}\right) \rightarrow H_{v} \mid v \in V\right\}
$$

where $C \downarrow_{v}$ is the set of literals of $C$ over atomic predicates occurring in $\Theta(v)$, and $H_{v}=\perp$ if $v=v_{r t}$ and $H_{v}=Q\left(\bar{x}_{Q}\right)$ if $v$ is a predicate variable $Q$. We pass $\mathcal{H}_{\text {thy }(C, V, E, \Theta)}$ to $\mathcal{A E}_{L_{\text {base }}}$ to decide if it has a $L_{\text {base }}$ restricted solution, and if so, we set the obtained solution as the partial tree interpolant for the theorylevel resolution proof nodes where $C$ occurs as the theory lemma. Otherwise, we can safely reject that the whole problem as having no $L_{b a s e}$-restricted tree interpolant and return noproof. To generate the tree interpolant for the whole, we adopt the proof-based approach that builds the tree interpolant in a bottom up manner following the rules shown in Figure $5 .{ }^{9}$ Here, outs $(v)$ is the set of atomic predicates occurring outside of the subtree rooted at $v$ (i.e., outs $(v)=$ $\left\{p \mid p\right.$ occurs in $\Theta\left(v^{\prime}\right)$ where $\left.\left.\left(v^{\prime}, v\right) \notin E^{*}\right\}\right)$, and $C \uparrow_{v}$ is the set of literals of $C$ over the atomic predicates occurring outside of the subtree rooted at $v$ (i.e., $C \uparrow_{v}=\{p \in C \mid p \in \operatorname{outs}(v)\} \cup\{\neg p \in C \mid p \in$ outs $\left.(v)\}\right)$. The rules HYP for clauses in the input tree and RES for resolution steps extend the analogous rules from the standard proof-based interpolation [12] to tree interpolation. As described above, THY uses the $L_{b a s e}$-restricted solution computed by the exact solver for

\footnotetext{
${ }^{9}$ We assume that each $\Theta(v)$ is CNF (if not, they can be transformed so via the Tseitin transformation [22]).
} 
the partial tree interpolant. As we show in Theorems 4 and 5 below, this achieves the desired relaxed $\left(L_{\text {base }}, L_{\text {base }}{ }^{\wedge \vee}\right)$-restricted tree interpolation.

First, we show that any tree interpolant obtained by the method is restricted to $L_{\text {base }} \wedge^{\wedge}$.

Theorem 4. Let $\mathcal{H}$ be a tree-like HCCS and $(V, E, \Theta)$ be the corresponding tree interpolation problem. Suppose $(V, E, \Theta) \vdash i t p \perp: I$. Then, $I$ is a tree interpolant of $(V, E, \Theta)$, and for all $P \in \operatorname{pvs}(\mathcal{H}), \lambda \bar{x}_{P} . I(P) \in L_{\text {base }}{ }^{\wedge}$.

Next, we prove that if there is a $L_{b a s e}$-restricted tree interpolant for the given tree interpolation instance, then the method infers some tree interpolant (and by Theorem 4 above, such a tree interpolant will be restricted to $L_{\text {base }}{ }^{\wedge \vee}$ ).

Theorem 5. Let $\mathcal{H}$ be a tree-like HCCS and $(V, E, \Theta)$ be the corresponding tree interpolation problem. Suppose there is a tree interpolant $I$ of $(V, E, \Theta)$ such that for all $P \in \operatorname{pvs}(\mathcal{H}), \lambda \bar{x}_{P} . I(P) \in L_{\text {base }}$. Then, $(V, E, \Theta) \vdash_{i t p} \perp: I^{\prime}$ for some $I^{\prime}$.

We note that $\mathcal{H}_{t h y(C, V, E, \Theta)}$ is always a conjunctive HCCS and is often much smaller than the input HCCS. Therefore, the expensive exact $L_{b a s e}$-restricted constraint solver $\mathcal{A E}_{L_{\text {base }}}$ is only applied to small conjunctive HCCSs, thereby making its job easier. Also, we note that, while we have presented RIxSolveA to be parameterized by an exact $L_{b a s e}$-restricted constraint solver passed as module, the algorithm actually works even if a relaxed $\left(L_{\text {base }}, L_{\text {ext }}\right)$-restricted constraint solver is used in place of the exact $L_{b a s e}$-restricted constraint solver. ${ }^{10}$ Therefore, RIxSolveA can actually be parameterized by RIxSolveA itself, but the solvers must be "primed" by some exact solver (e.g., RIxSolveA[RIxSolveA $\left.\left[\mathcal{A E}_{L_{\text {base }}}\right]\right]$ ).

Minimizing Theory Lemmas. When $\neg C$ is given as a possible propositional model by the SMT solver, we use the exact finite-language-restricted constraint solver $\mathcal{A E}_{L_{\text {base }}}$ to find a solution for $\mathcal{H}_{t h y(C, V, E, \Theta)}$. But, using $C$ directly as the theory lemma after $\mathcal{A E}_{L_{\text {base }}}$ finds a solution could result in the SMT solver producing many propositional models and lead to bad performance. (This is analogous to using $C$ directly as the theory lemma in an ordinary lazy SMT solving when the theory solver finds $\neg C$ unsatisfiable.) Instead, we let $\mathcal{A E}_{L_{\text {base }}}$ return the subset of the literals of $C$ that it used to find the solution, and use it to obtain a smaller theory lemma. (We refer to Appendix E for more detail.)

\subsection{The Constraint Solver RIxSolveB}

$\mathrm{RIxSolveB}$ is a relaxed $\left(L_{\text {base }}, \mathrm{LB}\left(L_{\text {base }} \cup L_{\text {ext }}, \mathcal{A \mathcal { U }}, \ell\right)\right)$-restricted constraint solver which takes as module a relaxed $\left(L_{\text {base }}, L_{\text {ext }}\right)$-restricted constraint solver, an unrestricted constraint solver $\mathcal{A U}$, and a positive integer parameter $\ell$. $\operatorname{LB}(L, \mathcal{A U}, \ell)$ is a finite language of predicates determined by $L, \mathcal{A U}$, and $\ell$.

We informally describe RIxSolveB. We select some fraction of predicate variables in a certain "fair" manner based on the parameter $\ell$ and use the relaxed

${ }^{10}$ More precisely, it becomes a relaxed $\left(L_{b a s e}, L_{e x t} \wedge \vee\right.$ )-restricted constraint solver when passed a relaxed $\left(L_{\text {base }}, L_{\text {ext }}\right)$-restricted constraint solver. 
$\left(L_{\text {base }}, L_{\text {ext }}\right)$-restricted solver provided as a module to look for solutions to just the selected predicate variables. After restricted solutions are obtained for the selected predicate variables, we use $\mathcal{A U}$ to look for unrestricted solutions to the remaining predicate variables, and return the combined solution as the solution for the input HCCS. Note that this technique reduces the number of predicate variables that the given relaxed $\left(L_{b a s e}, L_{e x t}\right)$-restricted solver needs to solve for, and therefore can be used to improve the performance of a relaxed $\left(L_{\text {base }}, L_{\text {ext }}\right)$ restricted solver. The key observation we use here is that HCCSs solved in a refinement process are all unwound instances of a fixed "generator" HCCS (i.e., $\left.\mathcal{H}_{\text {gen }(M)}\right)$. As we shall show next, the observation can be used to guarantee that the result is a relaxed finite-language-restricted solver, when the predicate variable selection is done in a certain proper way.

We describe the constraint solving algorithm in detail. In what follows, we extend the definition of a tree-like HCCS (cf. Section 3.1) so that the root clause can be a Horn clause whose head is of the form $P(\bar{x})$ where $P$ does not occur anywhere else in the HCCS. For such an HCCS $\mathcal{H}$, we say that $P$ is the root of $\mathcal{H}$ and write $\operatorname{root}(\mathcal{H})=P(\operatorname{root}(\mathcal{H})=\perp$ for $\mathcal{H}$ with a $\perp$-head root clause $)$.

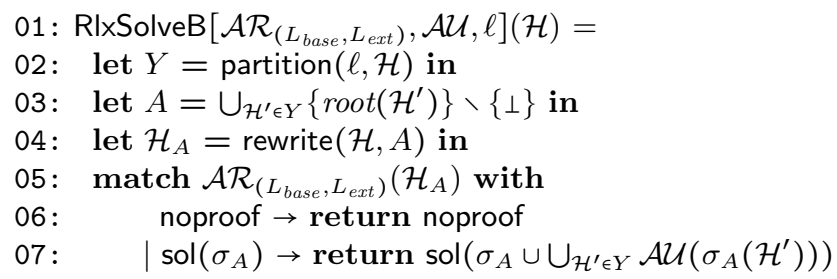

Fig. 6. The overview of RIxSolveB.

Let RIxSolveB be parameterized by the relaxed $\left(L_{b a s e}, L_{e x t}\right)$-restricted constraint solver $\mathcal{A R}_{\left(L_{\text {base }}, L_{\text {ext }}\right)}$, the unrestricted constraint solver $\mathcal{A U}$, and the positive integer $\ell$. Figure 6 shows the overview of RIxSolveB. RIxSolveB first partitions the input HCCS $\mathcal{H}$ into a set of tree-like HCCSs of depth at most $\ell$ in a top-down manner (line 2). Formally, partition is defined as follows.

$$
\begin{aligned}
& \text { partition }(\ell, \mathcal{H})= \\
& \text { if } \operatorname{depth}(\mathcal{H}) \leq \ell \text { then }\{\mathcal{H}\} \\
& \text { else let } \mathcal{H}^{\prime}, X=\operatorname{subtrees}(\ell, \mathcal{H}) \text { in } \\
& \quad\left\{\mathcal{H}^{\prime}\right\} \cup \cup_{\mathcal{H} \in X} \text { partition }(\ell, \mathcal{H})
\end{aligned}
$$

Here, subtrees $(\ell, \mathcal{H})$ returns the pair $\left(\mathcal{H}^{\prime}, X\right)$ such that 1.) $\mathcal{H}^{\prime}$ is the largest treelike subset of $\mathcal{H}$ containing the root clause of $\mathcal{H}$ and $\operatorname{depth}\left(\mathcal{H}^{\prime}\right)=\ell$, and 2.) $X$ is the set of subtrees of $\mathcal{H}$ rooted at each leaf of $\mathcal{H}^{\prime}$. It is easy to see that $Y$ partitions $\mathcal{H}$ (i.e., $\mathcal{H}=\bigcup Y$ and $\forall \mathcal{H}_{1}, \mathcal{H}_{2} \in Y$. $\mathcal{H}_{1} \neq \mathcal{H}_{2} \Rightarrow \mathcal{H}_{1} \cap \mathcal{H}_{2}=\varnothing$ ), and that each $\mathcal{H}^{\prime} \in Y$ is a tree-like HCCS of depth at most $\ell$. In fact, the partition is the coarsest of such partitions, where the only HCCSs in the partition having depth 
less than $\ell$ are the ones whose leaf predicate variables do not appear anywhere else in the partition.

By construction, only the root predicate variables are shared by different HCCSs in $Y$ (more precisely, a root of one HCCS appears as a leaf in another HCCS). RIxSolveB selects these shared predicate variables to be the ones to infer restricted solutions (line 3 ). It can be seen that the fraction of the selected predicate variables, that is $|A| /|\operatorname{pvs}(\mathcal{H})|$, is inversely proportional to the size of a depth $\ell$ tree-like subset of $\mathcal{H}$, and decreases rapidly as $\ell$ is increased.

To infer restricted solutions to $A$, RIxSolveB constructs the HCCS $\mathcal{H}_{A}$ such that $\operatorname{pvs}\left(\mathcal{H}_{A}\right)=A$, and solutions of $\mathcal{H}_{A}$ correspond exactly to the solutions of $\mathcal{H}$ restricted to $A$ (i.e., $\sigma \vDash \mathcal{H}_{A}$ if and only if $\exists \sigma^{\prime} . \sigma^{\prime} \uparrow_{A}=\sigma \wedge \sigma^{\prime} \vDash \mathcal{H}$ ). This is done by the operation rewrite $(\mathcal{H}, A)$ (line4), defined to be the application of the following rewriting relation $\rightarrow$ to $\mathcal{H}$ until convergence.

$$
\mathcal{H}^{\prime} \cup\left\{\Phi_{1} \rightarrow P(\bar{x}), \Phi_{2} \wedge P(\bar{y}) \rightarrow H\right\} \rightarrow \mathcal{H}^{\prime} \cup\left\{\Phi_{2} \wedge \Phi_{1}[\bar{y} / \bar{x}] \rightarrow H\right\}
$$

Here, $P \in \operatorname{pvs}(\mathcal{H}) \backslash A$, and $\Phi_{i}$ 's range over Horn clause bodies. (We assume that each Horn clause in $\mathcal{H}$ is over disjoint variables. Otherwise, we transform $\mathcal{H}$ into such a form by variable renaming.)

Then, RIxSolveB calls $\mathcal{A} \mathcal{R}_{\left(L_{b a s e}, L_{e x t}\right)}$ to find a $L_{\text {base }} \cup L_{\text {ext }}$-restricted solution for $\mathcal{H}_{A}$ (line 5$)$. If $\mathcal{A R}_{\left(L_{\text {base }}, L_{\text {ext }}\right)}$ returns noproof then no $L_{\text {base }}$ solution exists for $\mathcal{H}_{A}$ by the property of $\mathcal{A R}_{\left(L_{\text {base }}, L_{e x t}\right)}$, and by the construction above, it can be shown that no $L_{b a s e}$ solution exists for the input HCCS $\mathcal{H}$ either, and we safely return noproof (line 6) (see Theorem 7 for the proof). Otherwise, we obtain a solution $\sigma_{A}$ for $\mathcal{H}_{A}$, and RlxSolveB calls $\mathcal{A U}$ on each element of the partition with the solution $\sigma_{A}$ substituted (i.e., $\mathcal{A U}\left(\sigma_{A}\left(\mathcal{H}^{\prime}\right)\right.$ ) for each $\left.\mathcal{H}^{\prime} \in Y\right) .{ }^{11}$ This gives solutions for the remaining predicate variables in $\mathcal{H}$, and we return the union of $\sigma_{A}$ and these solutions as the final solution (line 7).

We argue that the produced solution is indeed a solution of $\mathcal{H}$. Let $Y=$ $\left\{\mathcal{H}_{1}, \ldots, \mathcal{H}_{n}\right\}$. Note that the only predicate variables shared by different elements in $Y$ are $A$. Therefore, $\sigma_{A}\left(\mathcal{H}_{1}\right), \ldots, \sigma_{A}\left(\mathcal{H}_{n}\right)$, and $\mathcal{H}_{A}$ are over disjoint predicate variables, and their solutions are over disjoint domains. Then, because $Y$ partitions $\mathcal{H}$, it follows that $\sigma_{A} \cup \cup_{\mathcal{H}^{\prime} \in Y} \mathcal{A} \mathcal{U}\left(\sigma_{A}\left(\mathcal{H}^{\prime}\right)\right)$ is a solution of $\mathcal{H}$.

To show that RIxSolveB is a relaxed finite-language-restricted constraint solver, it remains to show that the obtained solution is restricted to a finite language of predicates. We show that it is restricted to $\operatorname{LB}\left(L_{\text {base }} \cup L_{\text {ext }}, \mathcal{A U}, \ell\right)$ which is defined as follows. Let $L$ be a finite language. We define $\operatorname{LB}(L, \mathcal{A U}, \ell)$ as follows.

$$
\begin{aligned}
& \operatorname{LB}(L, \mathcal{A U}, \ell)=L \cup \cup_{\mathcal{H}^{\prime} \in X} \operatorname{ran}\left(\mathcal{A U}\left(\mathcal{H}^{\prime}\right)\right) \\
& \text { where } \\
& \quad X=\left\{\sigma\left(\mathcal{H}^{\prime}\right) \mid \mathcal{H}^{\prime} \in \operatorname{unwds}\left(\ell, \mathcal{H}_{\text {gen }(M)}\right) \text { and } \sigma \geq_{L} \mathcal{H}^{\prime}\right\}
\end{aligned}
$$

Here, $M$ is the program being verified (i.e., the input to the top-level procedure $\mathrm{Rl} \times$ Cegar), unwds $(\ell, \mathcal{H})$ is the set of unwound instances of $\mathcal{H}$ of depth at most $\ell$,

${ }^{11}$ Here, we extend the notion of substitution so that the result is tree-like: for $P \in$ $\operatorname{dom}\left(\sigma_{A}\right), \sigma_{A}(\Phi \rightarrow P(\bar{x}))=\neg \sigma_{A}(P)[\bar{x} / \tilde{\nu}(P)] \wedge \sigma_{A}(\Phi) \rightarrow \perp$. 
and $\sigma \geq_{L} \mathcal{H}$ if and only if $\sigma$ is a map from the leaves and the root of $\mathcal{H}$ to the predicates in $L$ (i.e., $\operatorname{dom}(\sigma)=$ leaves $(\mathcal{H}) \cup\{\operatorname{root}(\mathcal{H})\} \backslash\{\perp\}$ and $\operatorname{ran}(\mathcal{H}) \subseteq L)$. Note that $\mathcal{H}_{\text {gen }(M)}$ is a constant for the entire run of $\operatorname{Rlx} \operatorname{Cegar}(M)$, and unwds $(\ell, \mathcal{H})$ is finite for any $\mathcal{H}$ and $\ell$. Therefore, $\operatorname{LB}(L, \mathcal{A U}, \ell)$ is a finite language that is determined by $L, \mathcal{A U}$ and $\ell$.

We formally prove that RIxSolveB is indeed a relaxed $\left(L_{\text {base }}, \operatorname{LB}\left(L_{\text {base }} \cup\right.\right.$ $\left.L_{\text {ext }}, \mathcal{A U}, \ell\right)$ )-restricted constraint solver. First, we prove that the solution returned is indeed a solution of the input HCCS and that it is restricted to

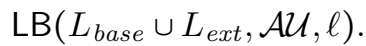

Theorem 6. Suppose $R / x$ Solve $B\left[\mathcal{A} \mathcal{R}_{\left(L_{b a s e}, L_{\text {ext }}\right)}, \mathcal{A U}, \ell\right](\mathcal{H})$ returns sol $(\sigma)$. Then, $\sigma \vDash \mathcal{H}$ and $\operatorname{ran}(\sigma) \subseteq L B\left(L_{\text {base }} \cup L_{\text {ext }}, \mathcal{A \mathcal { U }}, \ell\right)$.

Next, we show that some solution is returned if there exists a $L_{\text {base-restricted }}$ solution to the given HCCS (and by Theorem 6, such a solution is restricted to $\left.\operatorname{LB}\left(L_{\text {base }} \cup L_{\text {ext }}, \mathcal{A} \mathcal{U}, \ell\right)\right)$.

Theorem 7. Suppose that there exists $\sigma$ such that $\sigma \vDash \mathcal{H}$ and $\operatorname{ran}(\sigma) \subseteq L_{\text {base }}$. Then, RIxSolveB $\left[\mathcal{A R} \mathcal{R}_{\left(L_{b a s e}, L_{\text {ext }}\right)}, \mathcal{A U}, \ell\right](\mathcal{H})$ infers some $\sigma^{\prime}$ such that $\sigma^{\prime} \vDash \mathcal{H}$.

Example 6. Recall the HCCS $\mathcal{H}_{\text {exa }}$ from Example 3. Running RIxSolveB on $\mathcal{H}_{\text {exa }}$ with $\ell=2$, we have $Y=\operatorname{partition}\left(2, \mathcal{H}_{\text {exa }}\right)=\left\{\mathcal{H}_{1}, \mathcal{H}_{2}, \mathcal{H}_{3}\right\}$ where

$$
\begin{aligned}
& \mathcal{H}_{1}=\left\{\theta_{\mathrm{p} 3} \wedge Q_{1}(\bar{x}) \rightarrow Q_{2}\left(\bar{x}^{\prime}\right), \theta_{\mathrm{p} 4} \wedge Q_{2}(\bar{x}) \rightarrow \perp\right\} \\
& \mathcal{H}_{2}=\left\{\theta_{\mathrm{p} 2} \wedge P_{1}(\bar{x}) \rightarrow P_{2}\left(\bar{x}^{\prime}\right), P_{2}(\bar{x}) \rightarrow Q_{1}(\bar{x})\right\} \\
& \mathcal{H}_{3}=\left\{\theta_{\mathrm{p} 1} \rightarrow P_{1}(\bar{x})\right\}
\end{aligned}
$$

Then, the shared predicate variables that are selected to be restricted are $A=$ $\left\{\operatorname{root}\left(\mathcal{H}_{1}\right), \operatorname{root}\left(\mathcal{H}_{2}\right), \operatorname{root}\left(\mathcal{H}_{3}\right)\right\} \backslash\{\perp\}=\left\{P_{1}, Q_{1}\right\}$. And, $\mathcal{H}_{A}=\operatorname{rewrite}\left(\mathcal{H}_{\text {exa }}, A\right)$ is as shown below.

$$
\mathcal{H}_{A}=\left\{\theta_{\mathrm{p} 3} \wedge Q_{1}(\bar{x}) \wedge \theta_{\mathrm{p} 4}\left[\bar{x}^{\prime}, \bar{x}^{\prime \prime} / \bar{x}, \bar{x}^{\prime}\right] \rightarrow \perp, \theta_{\mathrm{p} 2} \wedge P_{1}(\bar{x}) \rightarrow Q_{1}\left(\bar{x}^{\prime}\right), \theta_{\mathrm{p} 1} \rightarrow P_{1}(\bar{x})\right\}
$$

where $\bar{x}^{\prime \prime}$ is a quintuple of fresh variables. RlxSolveB then calls $\mathcal{A R}_{\left(L_{\text {base }}, L_{\text {ext }}\right)}$ on $\mathcal{H}_{A}$ to obtain a restricted solution for $A$. Suppose the returned solution is $\sigma_{A}=\left\{P \mapsto a=b \Rightarrow y=z+x \mid P \in\left\{P_{1}, Q_{1}\right\}\right\}$. Then, $\sigma_{A}$ is applied to each element of the partition and we obtain $\sigma_{A}\left(\mathcal{H}_{1}\right), \sigma_{A}\left(\mathcal{H}_{2}\right)$, and $\sigma_{A}\left(\mathcal{H}_{3}\right)$ shown below.

$$
\begin{aligned}
& \sigma_{A}\left(\mathcal{H}_{1}\right)=\left\{\theta_{\mathrm{p} 3} \wedge(a=b \Rightarrow y=x+z) \rightarrow Q_{2}\left(\bar{x}^{\prime}\right), \theta_{\mathrm{p} 4} \wedge Q_{2}(\bar{x}) \rightarrow \perp\right\} \\
& \sigma_{A}\left(\mathcal{H}_{2}\right)=\left\{\theta_{\mathrm{p} 2} \wedge(a=b \Rightarrow y=x+z) \rightarrow P_{2}\left(\bar{x}^{\prime}\right), a=b \wedge y \neq x+z \wedge P_{2}(\bar{x}) \rightarrow \perp\right\} \\
& \sigma_{A}\left(\mathcal{H}_{3}\right)=\left\{a=b \wedge y \neq x+z \wedge \theta_{\mathrm{p} 1} \rightarrow \perp\right\}
\end{aligned}
$$

$\mathcal{A U}$ is called on $\sigma_{A}\left(\mathcal{H}_{1}\right), \sigma_{A}\left(\mathcal{H}_{2}\right)$, and $\sigma_{A}\left(\mathcal{H}_{3}\right)$ to infer unrestricted solutions to the remaining predicate variables. Suppose we have obtained the solutions $\sigma_{1}=\left\{Q_{2} \mapsto a=b \Rightarrow z \neq-1 \wedge y=x+z\right\}, \sigma_{2}=\left\{P_{2} \mapsto a=b \Rightarrow y=x+z\right\}$, and $\sigma_{3}=\varnothing$ for $\sigma_{A}\left(\mathcal{H}_{1}\right), \sigma_{A}\left(\mathcal{H}_{2}\right)$, and $\sigma_{A}\left(\mathcal{H}_{3}\right)$ respectively. Finally, RIxSolveB returns the combined map, $\sigma_{A} \cup \sigma_{1} \cup \sigma_{2} \cup \sigma_{3}$, as the solution inferred for the input HCCS $\mathcal{H}_{\text {exa }}$. 


\section{Implementation and Experiments}

We have implemented the new refinement algorithms RIxSolveA and RlxSolveB described in Section 3. The refinement algorithms require an exact finite-languagerestricted constraint solver and an unrestricted constraint solver to be provided as modules. An unrestricted constraint solver finds unrestricted solutions to the given tree-like HCCS. This is the ordinary constraint solving for tree-like HCCSs which is a well-studied problem, and we use the existing technique that iteratively solve the constraints one predicate variable at a time by using interpolation as a blackbox process (see $[23,5,20]$ for details). ${ }^{12}$

Exact Finite-Language-Restricted Constraint Solver. For the exact solver, we use a simple approach in which the finite predicate languages are represented by predicate templates containing unknowns of bounded range. We use an SMT solver ${ }^{13}$ to find an assignment to the unknowns within the bound that makes the templates into an actual solution. Below, we informally describe the process by an example, and defer the detailed description to Appendix E.

Example 7. Recall the program $M_{e x}$ from Example 1. Let $L_{b a s e}$ be the finite language of predicates consisting of conjunctions of at most two atomic predicates whose numeric constants are bounded in the range $\{-1,0,1\}$. We represent the language by the bounded predicate template shown below

$$
\begin{array}{r}
\lambda a, b, x, y, z . c_{1} a+c_{2} b+c_{3} x+c_{4} y+c_{5} z+c_{6} \leq 0 \wedge \\
c_{7} a+c_{8} b+c_{9} x+c_{10} y+c_{11} z+c_{12} \leq 0
\end{array}
$$

where $c_{i}$ 's are unknown constants each associated with the bound $\{-1,0,1\}$. Bounded predicate templates can concisely represent a finite language of predicates. ${ }^{14}$

Let $\xi$ be a bounded predicate template. To check if the given HCCS $\mathcal{H}$ has a solution in the language represented by $\xi$, we make a solution template $\sigma_{\xi}$ that maps each predicate variable in $\mathcal{H}$ to a copy of $\xi$ with fresh unknowns. Then, we check if there exists an assignment to the unknowns within the bounds that makes the solution template into an actual solution of $\mathcal{H}$, that is, we look for assignments to the unknowns within the bounds that satisfy $\forall h c \in \sigma_{\xi}(\mathcal{H})$. $\vDash h c$. For QFLRA, the latter can be done by applying the Motzkin's transposition theorem [18] to reduce the problem to the satisfiability problem for quantifierfree non-linear real arithmetic, and using an SMT solver to solve the resulting problem.

We note that the exact finite-language-restricted constraint solving is a highly expensive process and using it directly solve the whole HCCS is prohibitive. Indeed, as we show in the experiments, the exact solver fails to scale even on

\footnotetext{
${ }^{12}$ The implementation uses MathSAT 5 (http://mathsat.fbk.eu/) for the backend interpolation process.

13 The implementation uses Z3 (http://z3. codeplex.com/).

14 Note that such a language is generally not closed under conjunctions or disjunctions.
} 
relatively small constraint sets (see also the discussion in Section 5 and the complexity theoretic analysis in Appendix F).

Experiment Setup. We have experimented with the new refinement algorithms by using them in the refinement process of MoCHi [10]. MoCHi is a state-of-theart software model checker for higher-order functional programs based on predicate abstraction, CEGAR, and higher-order-recursion-scheme (HORS) model checking. MoCHi verifies assertion safety of OCaml programs. A verifier for functional programs such as $\mathrm{MoCHi}$ is suited for experimenting with the new refinement algorithm because Horn-clause constraints generated in such a verifier often contain non-trivial tree-like structure. (Intuitively, this is because the constraints express the flow of data in the program, and data often flow in a complex way in a functional program, e.g., passed to and returned from recursive functions, captured in closures, etc.)

The new refinement algorithms RIxSolveA and RlxSolveB are parametric. For this experiment, we parameterize them as follows to obtain a single refinement algorithm:

$$
\text { RIxSolveB }\left[\mathrm{RIxSolveA}\left[\mathcal{A E}_{L_{\text {base }}}\right], \mathcal{A U}, 4\right]
$$

Here, the exact $L_{\text {base }}$-restricted constraint solver $\mathcal{A E}_{L_{\text {base }}}$ and the unrestricted solver $\mathcal{A U}$ are the ones described above. That is, we use RlxSolveB parameterized to use as modules the relaxed $\left(L_{\text {base }},\left(L_{\text {base }}\right)^{\wedge \vee}\right)$-restricted constraint solver RIxSolveA (itself parameterized by the exact $L_{\text {base }}$-restricted constraint solver $\left.\mathcal{A E}_{L_{\text {base }}}\right)$ and the unrestricted constraint solver $\mathcal{A U}$, and with the parameter $\ell=4$. The strata of restricting predicate languages are built "dynamically" as the CEGAR iteration progresses, by starting from a small fixed $\left(L_{b a s e}^{0}, L_{e x t}^{0}\right)$ and enlarging the current $\left(L_{\text {base }}, L_{\text {ext }}\right)$ whenever the refinement algorithm returns noproof by using the unrestricted refinement process. ${ }^{15}$

We compare the new refinement algorithm with two other refinement methods: 1.) the ordinary (incomplete) unrestricted predicate search, and 2.) exact finite-language-restricted predicate search. The unrestricted predicate search algorithm is $\mathcal{A U}$, and the exact finite-language restricted predicate search algorithm is $\mathcal{A} \mathcal{E}$. For $\mathcal{A} \mathcal{E}$, we give (the $L_{\text {base }}$ part of) the same restricting predicate language given to the new algorithm when solving the corresponding HCCS.

We have ran the three refinement algorithms on 318 HCCSs generated by running MoCHi on 139 programs, measuring the time spent in each run of the refinement process. The benchmark programs are mostly taken from the previous work on MoCHi $[10,16,25,11]$. To obtain the benchmark HCCS set, we ran $\mathrm{MoCHi}$ on each benchmark program with the new refinement algorithm until completion or timeout and recorded the HCCS given as the input to each run of the refinement process. We also compare the overall verification speed of MoCHi when using the three refinement algorithms. This is done by running MoCHi with each of the refinement algorithm on the 139 benchmark programs. We have

${ }^{15}$ Formally, this is done by having a non-decreasing preorder of restricting predicate languages where the limit of any $\omega$-chain is $\mathcal{T}$, and when noproof is returned, the language raised to the least one containing the predicate inferred by $\mathcal{A} \mathcal{U}$. 
run the experiments on a machine with $2.69 \mathrm{GHz}$ i7-4600U processor with 16 GB of RAM, with the time limit of 100 seconds. The benchmark programs, the benchmark HCCSs and the experiment results data are available online [21].

Experiment Aim and Hypothesis. Because of the overhead from computing restricted proofs, we expect the individual refinement runs to be slower with the new refinement algorithm compared to an ordinary incomplete approach which only does unrestricted refinement, but faster than the more naïve complete approach that directly applies the exact finite-language-restricted proof search to the entire refinement problem. The main purpose of the experiment is to test this hypothesis. We also compare the overall verification speeds, but we do not expect a significant improvement on this aspect because of the inherent complexity of the verification problem. (For any sound and QFLRA-complete verifier, one can always find a program on which the verifier takes arbitrarily long time.)

Experiment Results and Analysis. Figure 7 shows the plots comparing the the run times of the new refinement algorithm (New Algorithm), the unrestricted refinement algorithm (Unrestricted), and the exact finite-language-restricted refinement algorithm (Exact) on each of the 318 benchmark HCCSs. As we have expected, the unrestricted refinement algorithm is the fastest of the three. The new algorithm performs quite competitively, however, and shows that it is able to achieve completeness with only a low overhead. Also, the plots show that the exact finite-language-restricted refinement algorithm is significantly slower, timing out on many instances that the other two algorithms were able to solve quickly.
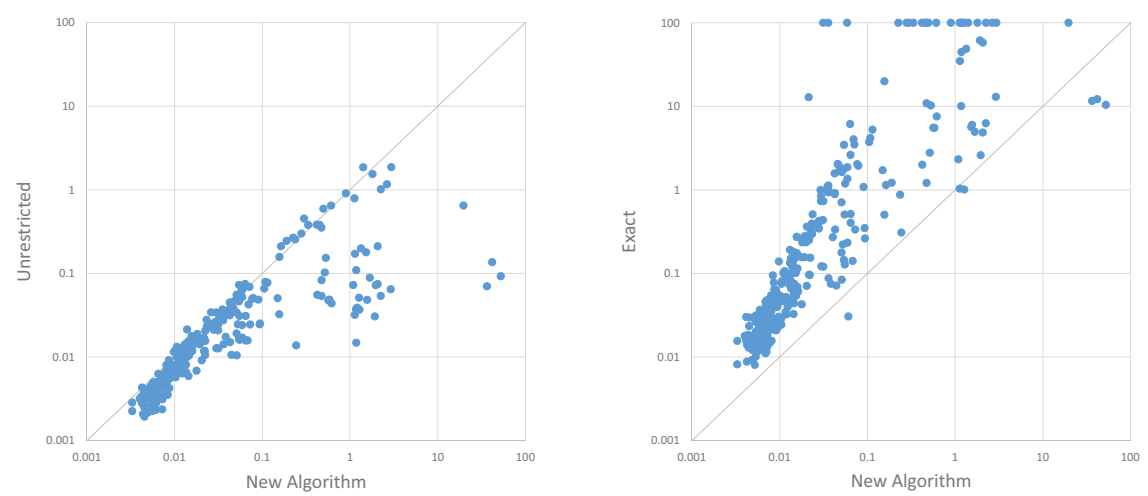

Fig. 7. Run time comparison of the refinement algorithms on benchmarks HCCSs

Figure 8 shows the plots comparing the run times of the overall verification process on each of the 139 benchmark programs for each refinement algorithm. 

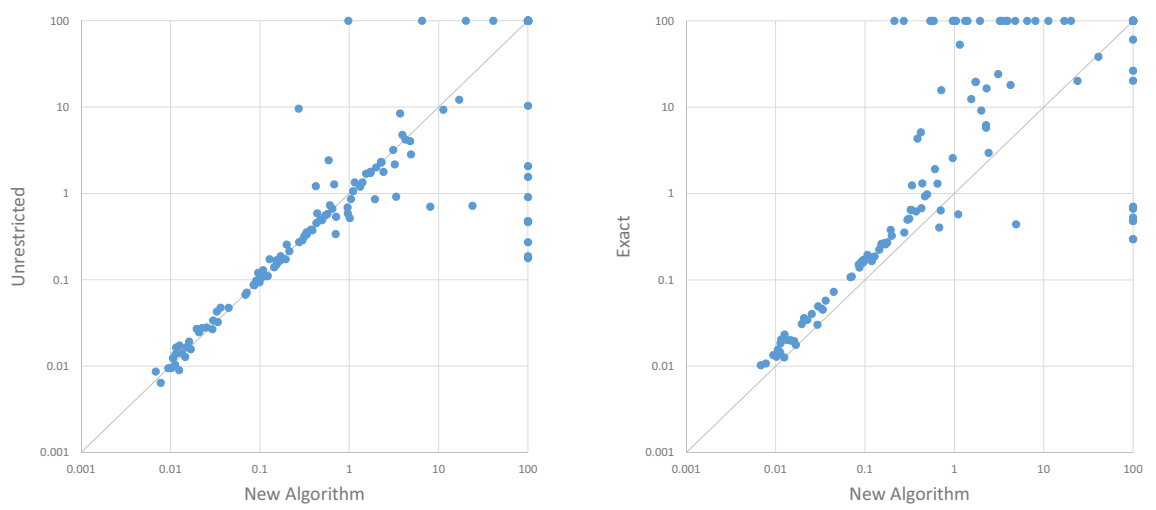

Fig. 8. Run time comparison of the refinement algorithms on benchmarks programs

The plots show that there is no clear winner in this comparison and none of the three outperformed the others on all benchmarks (while the unrestricted refinement edged out in the number of instances solved within the time limit, it also timed out on some instances the complete methods were able to solve). This is due to the inherent undecidability of the program verification problem, and the fact that the speed of overall verification depends heavily on subtle heuristic choices made by MoCHi. Such issues are largely outside of the scope of this paper, but they give interesting insights into what would be the good heuristics to use with the new refinement algorithm. For instance, an interesting behavior we have observed is that the stratified approaches (New Algorithm and Exact) sometimes infer more useless predicates than the ordinary unrestricted refinement because a stratified approach needs to add predicates to raise the language stratum till it reaches the level where a proof of the given program exists. Because MoCHi does eager predicate abstraction, its performance degrades exponentially in the number of predicates that are added to the candidate predicate set. This seems to have had a large negative impact on the stratified approaches. A possible way to address the issue maybe is to have MoCHi take a more lazy approach to predicate abstraction, or allow the language strata "coarseness" to be dynamically adjustable so that we can immediately jump to a large predicate language when it seems beneficial.

\section{Related Work}

Previous work $[8,13]$ has considered an exact stratification approach which requires the refinement process to exactly decide if a proof of the given counterexample's spuriousness exists in the current finite language stratum. As remarked before, an issue with exact stratification is the high cost of exact finitelanguage-restricted proof search. As we have shown empirically in Section 4, the exact finite-language-restricted proof search suffers from high overhead. (We 
also show complexity theoretic evidences for the inherent hardness of the exact search in Appendix F.) We note that relaxed stratification is a generalization of exact stratification. That is, exact stratification is a special case of relaxed stratification where $L_{\text {ext }}=\varnothing$.

We note that the interpolation technique that limits the theory-level reasoning to only emit restricted partial interpolants (cf. Section 3.2) has also been proposed in [8]. But, they target exact stratification and therefore requires the restricting language to be closed under conjunctions and disjunctions (so that $L^{\wedge \vee}=L$ ), which substantially reduces the applicability of the technique. ${ }^{16}$

\section{Conclusion}

We have presented a new approach to complete predicate refinement, called relaxed stratification, where the background theory is stratified into a sequence of finite predicate languages

$$
\left(L_{\text {base }}^{0}, L_{\text {ext }}^{0}\right),\left(L_{\text {base }}^{1}, L_{\text {ext }}^{1}\right), \ldots\left(L_{\text {base }}^{k}, L_{\text {ext }}^{k}\right), \ldots
$$

such that each run of the refinement process is restricted to only infer predicates from the current stratum $L_{\text {base }} \cup L_{\text {ext }}$. Contrary to previous approaches to complete refinement, the refinement process is neither required to decide the existence of a proof for the given counterexample in $L_{\text {base }} \cup L_{\text {ext }}$ nor in $L_{\text {base }}$, but is only required to return some proof if one exists in $L_{\text {base }}$. We have proved that the approach is complete despite the relaxed requirement, assuming that the strata of $L_{\text {base }}$ 's grow to eventually cover the predicates of the underlying theory. We have shown that the relaxed requirement can be used to build practical refinement algorithms that have low overhead and the completeness guarantee.

\section{References}

1. M. Colón, S. Sankaranarayanan, and H. Sipma. Linear invariant generation using non-linear constraint solving. In W. A. H. Jr. and F. Somenzi, editors, $C A V$, volume 2725 of Lecture Notes in Computer Science, pages 420-432. Springer, 2003.

2. S. Grebenshchikov, N. P. Lopes, C. Popeea, and A. Rybalchenko. Synthesizing software verifiers from proof rules. In J. Vitek, H. Lin, and F. Tip, editors, PLDI, pages 405-416. ACM, 2012.

3. A. Gupta, C. Popeea, and A. Rybalchenko. Predicate abstraction and refinement for verifying multi-threaded programs. In T. Ball and M. Sagiv, editors, POPL, pages 331-344. ACM, 2011.

${ }^{16}$ Contrary to $[8]$, in QFLRA, it is insufficient to only look for an atomic interpolant (i.e., a separating hyperplane) when interpolating between even just conjunctions of literals (i.e., polytopes) under a finite-language restriction. For example, consider interpolating between $y \leq 1 \wedge 2 x+y \leq-3 \wedge x \leq-1$ and $y+x \geq 1$ under the restriction that interpolants' constants are in $\{-1,0,1\}$. 
4. A. Gupta, C. Popeea, and A. Rybalchenko. Solving recursion-free horn clauses over LI+UIF. In H. Yang, editor, APLAS, volume 7078 of Lecture Notes in Computer Science, pages 188-203. Springer, 2011.

5. M. Heizmann, J. Hoenicke, and A. Podelski. Nested interpolants. In M. V. Hermenegildo and J. Palsberg, editors, POPL, pages 471-482. ACM, 2010.

6. T. A. Henzinger, R. Jhala, R. Majumdar, and K. L. McMillan. Abstractions from proofs. In N. D. Jones and X. Leroy, editors, POPL, pages 232-244. ACM, 2004.

7. R. Jhala and R. Majumdar. Software model checking. ACM Computing Surveys, 41(4), 2009.

8. R. Jhala and K. L. McMillan. A practical and complete approach to predicate refinement. In H. Hermanns and J. Palsberg, editors, TACAS, volume 3920 of Lecture Notes in Computer Science, pages 459-473. Springer, 2006.

9. D. Jovanovic and L. M. de Moura. Solving non-linear arithmetic. In B. Gramlich, D. Miller, and U. Sattler, editors, IJCAR, volume 7364 of Lecture Notes in Computer Science, pages 339-354. Springer, 2012.

10. N. Kobayashi, R. Sato, and H. Unno. Predicate abstraction and CEGAR for higher-order model checking. In M. W. Hall and D. A. Padua, editors, PLDI, pages 222-233. ACM, 2011.

11. T. Kuwahara, T. Terauchi, H. Unno, and N. Kobayashi. Automatic termination verification for higher-order functional programs. In Z. Shao, editor, ESOP, volume 8410 of Lecture Notes in Computer Science, pages 392-411. Springer, 2014.

12. K. L. McMillan. An interpolating theorem prover. Theoretical Computer Science, 345(1):101-121, 2005.

13. K. L. McMillan. Quantified invariant generation using an interpolating saturation prover. In C. R. Ramakrishnan and J. Rehof, editors, TACAS, volume 4963 of Lecture Notes in Computer Science, pages 413-427. Springer, 2008.

14. P. Rümmer, H. Hojjat, and V. Kuncak. Classifying and solving horn clauses for verification. In E. Cohen and A. Rybalchenko, editors, VSTTE, volume 8164 of Lecture Notes in Computer Science, pages 1-21. Springer, 2013.

15. P. Rümmer, H. Hojjat, and V. Kuncak. Disjunctive interpolants for Horn-clause verification. In N. Sharygina and H. Veith, editors, CAV, volume 8044 of Lecture Notes in Computer Science, pages 347-363. Springer, 2013.

16. R. Sato, H. Unno, and N. Kobayashi. Towards a scalable software model checker for higher-order programs. In E. Albert and S. Mu, editors, PEPM, pages 53-62. ACM, 2013.

17. M. Schaefer and C. Umans. Completeness in the polynomial-time hierarchy: A compendium. SIGACT news, 33(3):32-49, 2002.

18. A. Schrijver. Theory of linear and integer programming. Wiley, 1998.

19. R. Sebastiani. Lazy satisability modulo theories. JSAT, 3(3-4):141-224, 2007.

20. T. Terauchi. Dependent types from counterexamples. In M. V. Hermenegildo and J. Palsberg, editors, POPL, pages 119-130. ACM, 2010.

21. T. Terauchi and H. Unno. Relaxed stratification: A new approach to practical complete predicate refinement. 2015. http://www.jaist.ac.jp/ terauchi.

22. G. S. Tseitin. On the complexity of derivation in propositional calculus. Studies in constructive mathematics and mathematical logic, 2(115-125):10-13, 1968.

23. H. Unno and N. Kobayashi. Dependent type inference with interpolants. In A. Porto and F. J. López-Fraguas, editors, PPDP, pages 277-288. ACM, 2009.

24. H. Unno and T. Terauchi. Inferring simple solutions to recursion-free horn clauses via sampling. In $T A C A S, 2015$. To appear. 
25. H. Unno, T. Terauchi, and N. Kobayashi. Automating relatively complete verification of higher-order functional programs. In R. Giacobazzi and R. Cousot, editors, POPL, pages 75-86. ACM, 2013.

\section{A Proof of Theorem 1}

The following lemma states that the candidates returned by the refinement process must contain a new predicate.

Lemma 1 (Progress). Let $\operatorname{Abs}(M, F)=\operatorname{cex}\left(M^{\prime}\right)$ and $R / x \operatorname{Ref}\left(M^{\prime}, L_{\text {base }}, L_{\text {ext }}\right)=$ $\operatorname{prf}\left(F^{\prime}\right)$. Then, $F^{\prime} \nsubseteq F$.

Proof. Because RlxRef $\left(M^{\prime}, L_{\text {base }}, L_{\text {ext }}\right)=\operatorname{prf}\left(F^{\prime}\right)$, Abs $\left(M^{\prime}, F^{\prime}\right)=$ safe. Also, because $\operatorname{Abs}(M, F)=\operatorname{cex}\left(M^{\prime}\right), \operatorname{Abs}\left(M^{\prime}, F\right) \neq$ safe. Therefore, $F \neq F^{\prime}$, and by the monotonicity of Abs, it follows that $F^{\prime} \nsubseteq F$.

Theorem 1 (Completeness). If $\exists F \subseteq \mathcal{T}$.Abs $(M, F)=$ safe, then RlxCegar $(M)$ terminates and returns safe.

Proof. Let $F \subseteq \mathcal{T}$ be such that $\operatorname{Abs}(M, F)=$ safe. Because $\operatorname{Abs}(M, F)=$ safe, for any counterexample $M^{\prime}$ of $M$ (i.e., $\operatorname{Abs}\left(M, F^{\prime}\right)=\operatorname{cex}\left(M^{\prime}\right)$ for some $\left.F^{\prime}\right)$, $\operatorname{RIxRef}\left(M^{\prime}, L_{\text {base }}, L_{\text {ext }}\right) \neq$ unsafe for any $\left(L_{\text {base }}, L_{\text {ext }}\right)$. Therefore, it suffices to show that RIxCegar $(M)$ terminates.

For contradiction, suppose that RIxCegar $(M)$ does not terminate. Let $j \in \omega$ be such that $F \subseteq L_{\text {base }}^{j}$ (by the definition of the restricting language strata, such $j$ exists). Let $\left(L_{b a s e}^{l v l(i)}, L_{e x t}^{l v l(i)}\right)$ be the restricting predicate language at the $i$-th CEGAR iteration. By Lemma 1, infinitely many predicates must have been returned as candidates. Therefore, at some iteration, the language stratum must have reached level $j$, because RIxRef at each $l v l(i)$-th language stratum is restricted to only infer predicates from the finite set $L_{b a s e}^{l v l(i)} \cup L_{e x t}^{l v l(i)}$.

By the fact that RIxRef refutes any counterexample refutable in the $L_{\text {base }}$ part of the given restricting language and the fact that $F$ is sufficient to refute any counterexample of $M$, for any counterexample $M^{\prime}$ of $M$, we have $\operatorname{RIxRef}\left(M^{\prime}, L_{\text {base }}^{j}, L_{\text {ext }}^{j}\right)=\operatorname{prf}\left(F^{\prime}\right)$ for some $F^{\prime}$. Therefore, by Lemma 1 and the finiteness of $L_{\text {base }}^{j} \cup L_{e x t}^{j}$, it follows that a sufficient proof of $M$ 's safety must have been inferred without further raising the language stratum, and we obtain a contradiction.

\section{B Proofs of Theorem 4 and Theorem 5}

Let ins $(v)$ be the atomic predicates occurring in the subtree rooted at $v$, that is, ins $(v)=\left\{p \mid p\right.$ occurs in $\Theta\left(v^{\prime}\right)$ where $\left.\left(v^{\prime}, v\right) \in E^{*}\right\}$. Let $C \downarrow_{v}=C \backslash C \uparrow_{v}$, that is, $C \downarrow_{v}$ are the literals of $C$ over atomic predicates not occurring outside of the subtree rooted at $v$. 
We state the following assumptions regarding the input tree interpolation instance and the theory lemmas, which can be enforced by making each atomic predicate in the input syntactically distinct. ${ }^{17}$

(A1) For $C$ a theory lemma, for each $p$ occurring in $C$, there is a unique $v \in V$ such that $p$ occurs in $\Theta(v)$.

(A2) For $C$ a theory lemma, for each $v \in V, \neg C \downarrow_{v} \Rightarrow \Theta(v)$.

(A3) For each $v, v^{\prime} \in V$ and $C \in \Theta(v), C \downarrow v^{\prime}=C$ if $\left(v, v^{\prime}\right) \in E^{*}$ and otherwise $C \downarrow v^{\prime}=\perp$.

(A1) says that theory lemmas are used only to "communicate" atomic predicates across nodes. This follows from the observation that a lazy SMT solver interprets the atomic predicates in the input tree interpolation instance as distinct propositional variables. (A2) is a consequence of the fact that the negation of a theory lemma is a possible satisfying assignment of $\wedge_{v \in V} \Theta(v)$ with the atomic predicates interpreted as distinct propositional variables.

The following lemma states that any atomic predicate occurring in the derivation is from the input tree interpolation instance.

Lemma 2. Let $(V, E, \Theta) \vdash_{i t p} C: I$. Then, for any $p$ occurring in $C$, and any $v \in V, p \in \operatorname{outs}(v)$ or $p \in \operatorname{ins}(v)$.

Proof. By induction on the derivation of $(V, E, \Theta) \vdash_{i t p} C: I$.

\section{B.1 Proof of Theorem 4}

Theorem 4. Let $\mathcal{H}$ be a tree-like HCCS and $(V, E, \Theta)$ be the corresponding tree interpolation problem. Suppose $(V, E, \Theta) \vdash_{i t p} \perp: I$. Then, I is a tree interpolant of $(V, E, \Theta)$, and for all $P \in \operatorname{pvs}(\mathcal{H}), \lambda \bar{x}_{P} . I(P) \in L_{\text {base }}{ }^{\wedge}$.

We define a $C$-partial tree interpolant to be a map $I$ from $V$ to formulas in $\mathcal{T}$ such that

(pitp-1) $I\left(v_{r t}\right)=\perp$ for the root node $v_{r t}$;

(pitp-2) for each $v \in V, \vDash \Theta(v) \wedge \neg C \downarrow_{v} \wedge \wedge_{\left(v^{\prime}, v\right) \in E} I\left(v^{\prime}\right) \Rightarrow I(v)$; and

(pitp-3) for each $v \in V, f v s(I(v)) \subseteq\left(\bigcup_{\left(v^{\prime}, v\right) \in E^{*}} f v s\left(\Theta\left(v^{\prime}\right)\right)\right) \cap\left(\bigcup_{\left(v^{\prime}, v\right) \notin E^{*}} f v s\left(\Theta\left(v^{\prime}\right)\right)\right)$.

Note that only the second condition, (pitp-2), differs from the definition of (nonpartial) tree interpolant. Essentially, the condition (pitp-2) is the corresponding condition from the tree interpolant definition except that the labeling $\Theta(v)$ is strengthened by $\neg C \downarrow_{v}$. Clearly, a $\perp$-partial tree interpolant is a tree interpolant. Therefore, Theorem 4 follows from the following lemma.

Lemma 3. Let $\mathcal{H}$ be a tree-like HCCS and $(V, E, \Theta)$ be the corresponding tree interpolation problem. Suppose $(V, E, \Theta) \vdash_{i t p} \perp: I$. Then, $I$ is a $C$-partial tree interpolant of $(V, E, \Theta)$, and $(*)$ for all $P \in \operatorname{pvs}(\mathcal{H}), \lambda \bar{x}_{P} . I(P) \in L_{\text {base }} \wedge$.

\footnotetext{
${ }^{17}$ In practice, we label the atomic predicate occurring in the different nodes of the input tree interpolation instance with disjoint sets of labels, and define $C \uparrow_{v}$ and $C \downarrow_{v}$ to distinguish the atomic predicates by the labels.
} 
Proof. By induction on the derivation of $\left(V_{\mathcal{H}}, E_{\mathcal{H}}, \Theta\right) \vdash_{i t p} C: I$. We prove by a case analysis on the last rule.

\section{Thy}

Conditions ( $)$, (pitp-1), and (pitp-3) are trivially satisfied. We show condition (pitp-2) for each $v$. By (A1), we have that $\neg C \downarrow_{v} \Rightarrow \neg C \downarrow_{v}$. Therefore, by Theorem 3 and the property of $\mathcal{A E}$, if $v$ is a predicate variable $P$, then

$$
\Theta(P) \wedge \neg C \downarrow_{P} \wedge \wedge_{(Q, P) \in E} \phi_{Q} \Rightarrow \phi_{P}
$$

And, for $v=v_{r t}$, we have

$$
\Theta\left(v_{r t}\right) \wedge \neg C \downarrow_{v_{r t}} \wedge \wedge_{\left(Q, v_{r t}\right) \in E} \phi_{Q} \Rightarrow \perp
$$

\section{Нур}

Conditions ( $\bullet$ ) and (pitp-1) are trivially satisfied. Condition (pitp-3) follows from (A3). We show condition (pitp-2) for each $v^{\prime}$. For $v^{\prime}$ such that $\left(v, v^{\prime}\right) \notin$ $E^{*}$, the condition is trivial. Let $\left(v, v^{\prime}\right) \in E^{*}$. Because $C \downarrow_{v^{\prime}} \vee C \uparrow_{v^{\prime}}=C$, the condition is equivalent to $\Theta\left(v^{\prime}\right) \wedge \wedge_{\left(v^{\prime \prime}, v^{\prime}\right) \in E} I\left(v^{\prime \prime}\right) \Rightarrow C$. If $v^{\prime}=v$, then the result follows from $\Theta(v) \Rightarrow C$. Otherwise, for some $v^{\prime \prime}$ such that $\left(v^{\prime \prime}, v^{\prime}\right) \in E$, Res we have that $I\left(v^{\prime \prime}\right)=C \uparrow_{v^{\prime \prime}}$, and the result follows from $C \uparrow_{v^{\prime \prime}} \Rightarrow C$.

Conditions (*), (pitp-1), and (pitp-3) follow immediately from induction hypothesis. We show condition (pitp-2) for each $v$. We split the argument on whether $p \in \operatorname{outs}(v)$ or not.

\section{Case $p \in$ outs $(v)$}

We have $I_{3}(v)=I_{1}(v) \wedge I_{2}(v)$. For any $v^{\prime}$ such that $\left(v^{\prime}, v\right) \in E$, we have $p \in$ outs $\left(v^{\prime}\right)$. Therefore, $I_{3}\left(v^{\prime}\right)=I_{1}\left(v^{\prime}\right) \wedge I_{2}^{\prime}\left(v^{\prime}\right)$ for any such $v^{\prime}$. Therefore, it suffices to show that

$$
\begin{aligned}
\Theta(v) \wedge \neg\left(C_{1} \vee C_{2}\right) \downarrow_{v} \wedge \wedge \wedge_{\left(v^{\prime}, v\right) \in E} & I_{1}\left(v^{\prime}\right) \wedge I_{2}\left(v^{\prime}\right) \\
& \Rightarrow I_{1}(v) \wedge I_{2}(v)
\end{aligned}
$$

By induction hypothesis, we have

$$
\Theta(v) \wedge \neg\left(p \vee C_{1}\right) \downarrow_{v} \wedge \wedge_{\left(v^{\prime}, v\right) \in E} I_{1}\left(v^{\prime}\right) \Rightarrow I_{1}(v)
$$

and,

$$
\Theta(v) \wedge \neg\left(\neg p \vee C_{2}\right) \downarrow_{v} \wedge \wedge_{\left(v^{\prime}, v\right) \in E} I_{2}\left(v^{\prime}\right) \Rightarrow I_{2}(v)
$$

Because $\left(p \vee C_{1}\right) \downarrow_{v}=C_{1} \downarrow_{v}$ and $\left(\neg p \vee C_{2}\right) \downarrow_{v}=C_{2} \downarrow_{v}$, the result follows.

\section{Case $p \notin o u t s(v)$}

We have $I_{3}(v)=I_{1}(v) \vee I_{2}(v)$. By Lemma 2, there is at most one $v^{\prime}$ such that $\left(v^{\prime}, v\right) \in E$ and $p \notin$ outs $\left(v^{\prime}\right)$. Let $v^{\prime}$ be such a node. (The argument is similar for the case when no such $v^{\prime}$ exists, i.e., when $p$ occurs in $\Theta(v)$.) Let

$$
V^{\prime \prime}=\left\{v^{\prime \prime} \mid\left(v^{\prime \prime}, v\right) \in E \text { and } p \in \operatorname{outs}\left(v^{\prime \prime}\right)\right\}
$$


Then, it suffices to show that

$$
\begin{aligned}
& \Theta(v) \wedge \neg\left(C_{1} \vee C_{2}\right) \downarrow_{v} \wedge \\
&\left(I_{1}\left(v^{\prime}\right) \vee I_{2}\left(v^{\prime}\right)\right) \wedge \wedge \wedge_{v^{\prime \prime} \in V^{\prime \prime}} I_{1}\left(v^{\prime \prime}\right) \wedge I_{2}\left(v^{\prime \prime}\right) \\
& \Rightarrow I_{1}(v) \vee I_{2}(v)
\end{aligned}
$$

By induction hypothesis, we have

$$
\Theta(v) \wedge \neg\left(p \vee C_{1}\right) \downarrow_{v} \wedge \wedge_{\left(v^{\prime}, v\right) \in E} I_{1}\left(v^{\prime}\right) \Rightarrow I_{1}(v)
$$

and,

$$
\Theta(v) \wedge \neg\left(\neg p \vee C_{2}\right) \downarrow_{v} \wedge \wedge_{\left(v^{\prime}, v\right) \in E} I_{2}\left(v^{\prime}\right) \Rightarrow I_{2}(v)
$$

Because $\left(p \vee C_{1}\right) \downarrow_{v}=p \vee C_{1} \downarrow_{v}$ and $\left(\neg p \vee C_{2}\right) \downarrow_{v}=\neg p \vee C_{2} \downarrow_{v}$, the result follows from the fact

$$
\begin{aligned}
\left(I_{1}\left(v^{\prime}\right) \vee I_{2}\left(v^{\prime}\right)\right) & \wedge \wedge_{v^{\prime \prime} \in V^{\prime \prime}} I_{1}\left(v^{\prime \prime}\right) \\
\left(\wedge_{\left(v^{\prime}, v\right) \in E} I_{1}\left(v^{\prime}\right)\right) & \wedge\left(I_{2}\left(v^{\prime \prime}\right) \Rightarrow\right. \\
\left(v^{\prime}, v\right) \in E & \left.I_{2}\left(v^{\prime}\right)\right)
\end{aligned}
$$

and a resolution on $p$.

\section{B.2 Proof of Theorem 5}

Theorem 5. Let $\mathcal{H}$ be a tree-like HCCS and $(V, E, \Theta)$ be the corresponding tree interpolation problem. Suppose there is a tree interpolant $I$ of $(V, E, \Theta)$ such that for all $P \in \operatorname{pvs}(\mathcal{H}), \lambda \bar{x}_{P} . I(P) \in L_{\text {base }}$. Then, $(V, E, \Theta) \vdash_{i t p} \perp: I^{\prime}$ for some $I^{\prime}$.

The theorem follows from the lemma below and the refutation completeness of resolution (for CNF).

Lemma 4. Let $\mathcal{H}$ be a tree-like HCCS and $(V, E, \Theta)$ be the corresponding tree interpolation problem. Suppose that there exists a tree interpolant I of $(V, E, \Theta)$ such that for all $P \in \operatorname{pvs}(\mathcal{H}), \lambda \bar{x}_{P} . I(P) \in L_{\text {base }}$. Suppose that $C$ is a theory lemma. Then, $(V, E, \Theta) \vdash_{i t p} C: I^{\prime}$ for some $I^{\prime}$.

Proof. We derive $(V, E, \Theta) \vdash_{i t p} C: I^{\prime}$ via THY. It suffices to show that $\sigma$ such that $\sigma(P)=\lambda \bar{x}_{P} . I(P)$ for each $P$ is a $L_{b a s e}$ solution of $\mathcal{H}_{t h y(C, V, E, \Theta)}$. By construction, $\operatorname{ran}(\sigma) \subseteq L_{\text {base }}$. Therefore, it suffices to show that $\vDash \sigma(h c)$ for each $h c \in \mathcal{H}_{t h y}(C, V, E, \Theta)$.

Because $I$ is a tree interpolant of $(V, E, \Theta)$, we have

$$
\neg \Theta\left(v_{r t}\right) \wedge \bigwedge_{\left(Q, v_{r t}\right) \in E} \sigma(Q) \Rightarrow \perp
$$

and for each $P$,

$$
\neg \Theta(P) \wedge \bigwedge_{\left(Q, v_{r t}\right) \in E} \sigma(Q) \Rightarrow \sigma(P)
$$


Therefore, by (A2), for the root clause of $\mathcal{H}_{t h y(C, V, E, \Theta)}$, we have

$$
\neg C \downarrow_{v_{r t}} \wedge \bigwedge_{\left(Q, v_{r t}\right) \in E} \sigma(Q) \Rightarrow \perp
$$

And, for each non-root clause in $\mathcal{H}_{t h y(C, V, E, \Theta)}$, we have

$$
\neg C \downarrow_{P} \wedge \bigwedge_{(Q, P) \in E} \sigma(Q) \Rightarrow \sigma(P)
$$

\section{Proof of Theorem 6}

Theorem 6. Suppose $R / x \operatorname{Solve} B\left[\mathcal{A} \mathcal{R}_{\left(L_{b a s e}, L_{e x t}\right)}, \mathcal{A U}, \ell\right](\mathcal{H})$ returns sol $(\sigma)$. Then, $\sigma \vDash \mathcal{H}$ and $\operatorname{ran}(\sigma) \subseteq L B\left(L_{\text {base }} \cup L_{\text {ext }}, \mathcal{A} \mathcal{U}, \ell\right)$.

Proof. The solution $\sigma$ inferred is of the form $\sigma_{A} \cup \bigcup_{i \in\{1, \ldots, n\}} \sigma_{i}$ where

- $\sigma_{A}$ is a solution of $\mathcal{H}_{A}$; and

- each $\sigma_{i}$ is a solution of $\sigma_{A}\left(\mathcal{H}_{i}\right)$ such that $\left\{\mathcal{H}_{1}, \ldots, \mathcal{H}_{n}\right\}=Y$ partitions the input HCCS $\mathcal{H}$.

By construction, we have that $\sigma_{A} \cup \sigma_{i} \vDash \mathcal{H}_{i}$ for each $\mathcal{H}_{i} \in Y$. Therefore, by disjointness of $\sigma_{A}$ and each $\sigma_{i}$, we have $\sigma \vDash \mathcal{H}$.

Next, we show that $\operatorname{ran}(\sigma) \subseteq \operatorname{LB}\left(L_{\text {base }} \cup L_{\text {ext }}, \mathcal{A} \mathcal{U}, \ell\right)$. For each $\mathcal{H}_{i}$, we have $\mathcal{H}_{i} \in$ unwds $\left(\ell, \mathcal{H}_{\text {gen }(M)}\right)$ and $\sigma_{A} \geq_{L_{\text {base }} \cup L_{\text {ext }}} \mathcal{H}_{i}$. Therefore, for each $\mathcal{H}_{i}$,

$$
\operatorname{ran}\left(\sigma_{i}\right)=\operatorname{ran}\left(\mathcal{A U}\left(\sigma_{A}\left(\mathcal{H}_{i}\right)\right)\right) \subseteq \bigcup_{\mathcal{H}^{\prime} \in X} \operatorname{ran}\left(\mathcal{A U}\left(\mathcal{H}^{\prime}\right)\right)
$$

where $X=\left\{\sigma\left(\mathcal{H}^{\prime}\right) \mid \mathcal{H}^{\prime} \in \operatorname{unwds}\left(\ell, \mathcal{H}_{\text {gen }(M)}\right)\right.$ and $\left.\sigma \geq_{L} \mathcal{H}^{\prime}\right\}$. Therefore, we have $\operatorname{ran}(\sigma) \subseteq \mathrm{LB}\left(L_{\text {base }} \cup L_{\text {ext }}, \mathcal{A} \mathcal{U}, \ell\right)$ because $\operatorname{ran}\left(\sigma_{A}\right) \subseteq L_{\text {base }} \cup L_{\text {ext }}$.

\section{Proof of Theorem 7}

Theorem 7. Suppose that there exists $\sigma$ such that $\sigma \vDash \mathcal{H}$ and $\operatorname{ran}(\sigma) \subseteq L_{\text {base }}$. Then, RIxSolveB $\left[\mathcal{A} \mathcal{R}_{\left(L_{b a s e}, L_{\text {ext }}\right)}, \mathcal{A U}, \ell\right](\mathcal{H})$ infers some $\sigma^{\prime}$ such that $\sigma^{\prime} \vDash \mathcal{H}$.

Proof. Let $Y, A$ and $\mathcal{H}_{A}$ be as constructed in Figure 6. Let $\sigma \uparrow_{A}$ be $\sigma$ restricted to $A$. Then, by construction, $\sigma \uparrow_{A} \vDash \mathcal{H}_{A}$. Therefore, $\mathcal{H}_{A}$ has a $L_{\text {base }}$ solution, and $\mathcal{A R}_{\left(L_{\text {base }}, L_{\text {ext }}\right)}\left(\mathcal{H}_{A}\right)$ returns some solution $\sigma_{A}$ of $\mathcal{H}_{A}$.

By construction, there exists $\sigma^{\prime} \supseteq \sigma_{A}$ such that $\sigma^{\prime} \vDash \mathcal{H}$. Therefore, each $\sigma_{A}\left(\mathcal{H}_{i}\right)$ for $\mathcal{H}_{i} \in Y$ is solvable because $\sigma^{\prime} \uparrow_{p v s\left(\sigma_{A}\left(\mathcal{H}_{i}\right)\right)} \vDash \sigma_{A}\left(\mathcal{H}_{i}\right)$. Then, because $\mathcal{A U}$ returns some solution of the given solvable HCCS, it follows that RIxSolveB returns some solution of $\mathcal{H}$. 


\section{E Exact $L$-restricted Constraint Solving for QFLRA}

A term $t$ in the signature of QFLRA is either a variable $x$ or an affine expression $a_{1} t_{1}+\cdots+a_{n} t_{n}+a_{n+1}$ where $a_{1}, \ldots, a_{n+1}$ are integer constants. We define a bounded term template to be an expression of the form $c^{b} t$ where $t$ is a term and $c^{b}$ is an bounded unknown constant annotated with a finite set $b \subseteq \mathbb{Z}$ as its bound. We define a bounded formula template to be a QFLRA formula that contain bounded term templates as (ordinary) terms. For example, $c_{1}^{\{0,1\}}+c_{2}^{\{1,2\}} x+c_{3}^{\{0,1\}} y \leq z$ is a bounded formula template. We define bounded predicate template to be of the form $\lambda \bar{x} . \phi$ where $\phi$ is a bounded formula template and $f v s(\phi) \subseteq\{\bar{x}\}$.

For a bounded predicate template $\xi$, we define $L_{\xi}$ to be the set of (nontemplate) predicates obtained by replacing each $c^{b}$ in $\xi$ by an integer $a \in b$. Clearly, $L_{\xi}$ is finite for any $\xi$.

We describe the exact $L_{\xi}$-restricted constraint solver $\mathcal{A E}_{L_{\xi}}$. It takes a treelike HCCS $\mathcal{H}$ as input and looks for a solution $\sigma$ such that $\sigma \vDash \mathcal{H}$ and $\operatorname{ran}(\sigma) \subseteq L_{\xi}$. $\mathcal{A E}_{L_{\xi}}$ does this as follows. First, by variable renaming, we transform $\mathcal{H}$ into a form such that each Horn clause is over disjoint variables. Then, we translate $\mathcal{H}$ to $\sigma_{\xi}(\mathcal{H})$ where $\sigma_{\xi}$ maps each predicate variable $P \in \operatorname{pvs}(S)$ to $\xi_{P}$ such that $\xi_{P}$ is a copy of $\xi$ with the unknowns replaced fresh unknowns with the same bounds. ${ }^{18}$

Note that $\sigma_{\xi}(S)$ has no predicate variable. Let $\Psi=\wedge \sigma_{\xi}(S) . \Psi$ is a bounded formula template, interpreting $\rightarrow$ as $\Rightarrow$. Let $c_{1}^{b_{1}}, \ldots, c_{n}^{b_{n}}$ be the bounded unknowns occurring in $\Psi$, and $\bar{x}$ be the variables occurring in $\Psi$. Now, to find an assignment to the unknowns that make the bounded formula templates into a solution of $\mathcal{H}$, it suffices to find an assignment to each $c_{i}^{b_{i}}$ within the bound $b_{i}$ that satisfy $\forall \bar{x} . \Psi$.

We convert $\Psi$ to an equivalent formula $\wedge_{i} \varphi_{i}$ via a CNF conversion so that each $\varphi_{i}$ is of the form $\neg(\boldsymbol{A} \bar{x} \leq \boldsymbol{a} \wedge \boldsymbol{B} \bar{x}<\boldsymbol{b})$ where $\boldsymbol{A}$ and $\boldsymbol{B}$ (resp. $\boldsymbol{a}$ and $\left.\boldsymbol{b}\right)$ are matrices (resp. vectors) whose elements are polynomials on $c_{1}^{b_{1}}, \ldots, c_{n}^{b_{n}}$. Then, we translate each $\varphi_{i}$ by applying Motzkin's transposition theorem [18]:

Theorem 8 (Motkin's transposition theorem). Consider the following system of linear inequalities over real-valued variables $\bar{x}$.

$$
\boldsymbol{A} \bar{x} \leq \boldsymbol{a} \wedge \boldsymbol{B} \bar{x}<\boldsymbol{b}
$$

The system is unsatisfiable if and only if there exist non-negative real-valued vectors $\boldsymbol{r}$ and $\boldsymbol{p}$ such that

$$
\boldsymbol{r} \boldsymbol{A}+\boldsymbol{p} \boldsymbol{B}=0 \wedge(\boldsymbol{r a}+\boldsymbol{p b}<0 \vee(\boldsymbol{p} \neq 0 \wedge \boldsymbol{r a}+\boldsymbol{p b} \leq 0))
$$

Applying the Motzkin's transposition theorem, the satisfiability of $\forall \bar{x} \cdot \wedge_{i} \varphi_{i}$ is reduced to that of $\Psi^{\prime}$ where $\Psi^{\prime}$ is a quantifier-free non-linear real arithmetic

\footnotetext{
${ }^{18}$ For simplicity, we assumes that the predicate variables in $\mathcal{H}$ have same arities. Otherwise, the restricting language is represented by a bounded predicate template per a predicate variable of different arities.
} 
formula on variables $\bar{r}, \bar{p}$, and $c_{1}^{b_{1}}, \ldots, c_{n}^{b_{n}}$ where the variables $\bar{r}$ and $\bar{p}$ are factor variables that are introduced in the translation. Finally, we conjunct $\Psi^{\prime}$ with the formula encoding the bound information for each $c_{i}^{b_{i}}$, and apply a decision procedure for quantifier-free non-linear real arithmetic [9] to find a satisfying assignment to the unknowns. If no satisfying assignments are found, then we return noproof. Otherwise, the assignments found for the unknowns $c_{1}^{b_{1}}, \ldots, c_{n}^{b_{n}}$ are substituted to $\sigma_{\xi}$ to obtain a solution for the input HCCS.

Note that, contrary to the template-based program verification [1] that use the templates directly as a solution for a recursive constraint set (i.e., one with cyclic $\sim \mathcal{H}$ if expressed as Horn-clause constraints), we use the templates to solve only recursion-free constraints. The role of templates here is only for restricting the solution to a finite language.

Returning Small Models to Minimize Theory Lemmas. Recall that the input to $\mathcal{A} \mathcal{E}$ given by RlxSolveA is a HCCS $\mathcal{H}_{C}$ that consists of Horn clauses of the form $\neg C \downarrow_{v} \wedge \wedge_{(Q, v) \in E} Q\left(\bar{x}_{Q}\right) \rightarrow H$ where $\neg C$ is the possible model found by the SMT solver (cf. Section 3.2). To minimize the model, we wish to find a small subset $C^{\prime} \subseteq C$ such that $\mathcal{H}_{C^{\prime}}$ is still $L_{\xi}$ solvable where $\mathcal{H}_{C^{\prime}}$ is $\mathcal{H}_{C}$ with each formula part of the body $\neg C \downarrow_{v}$ replaced with $\neg C^{\prime} \downarrow_{v}$. To this end, we solve $\mathcal{H}_{C}$ as described above and obtain a solution $\sigma$ for $\mathcal{H}_{C}$. Then, we check the assignment inferred for the factor variables $\bar{r}$ and $\bar{p}$ and remove from $C$ any literal whose associated factor has 0 inferred as the assignment (note that each literal of $C$ is associated with some row of $[\boldsymbol{A} \mid \boldsymbol{a}]$ or $[\boldsymbol{B} \mid \boldsymbol{b}]$ ) and return the resulting model along with $\sigma$. The correctness of the approach follows from the fact that such literals do not contribute to the solution $\sigma$ obtained for $\mathcal{H}_{C}$.

\section{F Computational Complexity of Exact Finite-Language- Restricted Predicate Search}

Formally, the decision version of exact finite-language-restricted predicate search is the problem of deciding, given a tree-like HCCS $S$ and $\operatorname{repl}(L)$ representing a finite language, whether there exists a solution for $S$ in $L$. We investigate the problem's computational complexity for QFLRA. ${ }^{19}$

First, we note that the unrestricted version of the problem is coNP-complete. This follows from the equivalence of tree-like HCCS solving and tree interpolation (cf. Section 3.2) and the fact that (the decision version of) tree interpolation for QFLRA is coNP-complete.

Next, we consider the case when $\operatorname{repl}(L)$ is given as a bounded predicate template as described in Section 4 . Then, the exact finite-language-restricted predicate search problem can be shown to be $\Sigma_{2}$-complete. The result shows a gap from the unrestricted case, assuming that the polynomial hierarchy does not collapse at the first level.

${ }^{19}$ A similar analysis can be applied to other FOL theories whose TAUT and (unrestricted) interpolation are coNP-complete. 
Theorem 9. The exact finite-language-restricted predicate search problem is $\Sigma_{2}$-complete for repl $(L)$ given as a bounded predicate template.

Proof.

In $\Sigma_{2}$

This is immediate from the fact that checking whether a given assignment $\sigma: p v s(S) \rightarrow L$ is a solution of $S$ is in coNP.

$\Sigma_{2}$-hard

We reduce from the $\Sigma_{2}$-complete problem of deciding if a quantified propositional formula of the form $\exists \bar{x} . \forall \bar{y} . \Phi$ is true, where $\Phi$ is a propositional formula on variables $\bar{x}, \bar{y}$. Let $\exists \bar{x} . \forall \bar{y} . \Phi$ be the given formula. Let $x_{1}, \ldots, x_{n}=\bar{x}$, and $y_{1}, \ldots, y_{m}=\bar{y}$ Let $c_{1}, \ldots, c_{n}=\bar{c}$ be unknowns each having the bound $\{0,1\}$. We construct a bounded predicate template $\operatorname{repl}(L)$ of the form $\lambda \bar{y} . \phi$ where $\phi$ is a bounded formula template on variables $\bar{y}$ and unknowns $\bar{c}$ such that for all $\overline{v_{x}} \in\{0,1\}^{n}$ and $\overline{v_{y}} \in \mathbb{R}^{m}, \neg \phi\left[\overline{v_{x}} / \bar{c}\right]\left[\overline{v_{y}} / \bar{y}\right]$ if and only if $\overline{v_{y}} \in\{0,1\}^{m}$ and $\Phi\left[\overline{v_{x}} / \bar{x}\right]\left[\overline{v_{y}} / \bar{y}\right]$. Formally, $\neg \phi$ can be constructed from $\Phi$ by replacing each variable occurrence $x_{i} \in \bar{x}$ (resp. $y_{i} \in \bar{y}$ ) by $c_{i}=1$ (resp. $y_{i}=1$ ), and conjuncting the formula $x_{i}=0 \vee x_{i}=1$ for each variable $x_{i} \in \bar{x}$. Let $S=\{P(\bar{y}) \rightarrow \perp\}$. Then, $S$ has a solution in $L$ if and only if $\exists \bar{x} . \forall \bar{y} . \Phi$ is true.

We note that, in general (for QFLRA), the problem of exact finite-languagerestricted predicate search is in $\Sigma_{2}$ for any language representation where the size of formulas in the language is polynomial in the size of the representation.

Next, we consider the case when $\operatorname{repl}(L)$ represents the maximum size of formulas in $L$. We consider three definitions of "formula size". We show that the problem is $\Sigma_{2}$-hard when the size is defined to be the number of literals in CNF or DNF, and is $\Sigma_{2}$-hard under Turing-reductions when the size is defined to be the number of literals (in arbitrary form). (Here, we assume that the constants appearing in $L$ are finitely bounded, so that $L$ is finite.)

Theorem 10. For $\operatorname{repl}(L)$ given as the size of the maximum formula in $L$, the exact finite-language-restricted predicate search problem is

- $\Sigma_{2}$-hard when the size is defined to be the number of literals in CNF;

- $\Sigma_{2}$-hard when the size is defined to be the number of literals in DNF; and

- $\Sigma_{2}$-hard under Turing-reductions when the size is defined to be the number of literals.

Proof. $\Sigma_{2}$-hardness for the CNF case is shown by a reduction from SHORT $\mathrm{CNF}$, and by a reduction from MIN DNF for the DNF case, and by a reduction from MINIMUM EQUIVALENT EXPRESSION for the general case [17]. The reduction in each case has the same structure: we use the observation that exact finite-language-restricted predicate search contains the problem of finding an equivalent formula in the restricting language. That is, checking if there exists a formula in $L$ equivalent to the given formula $\theta$ can be reduced to checking if the HCCS $\{\theta \rightarrow P(\bar{x}), P(\bar{x}) \wedge \neg \theta \rightarrow \perp\}$ has a solution in $L$ where $\bar{x}$ are the variables occurring in $\theta$. 